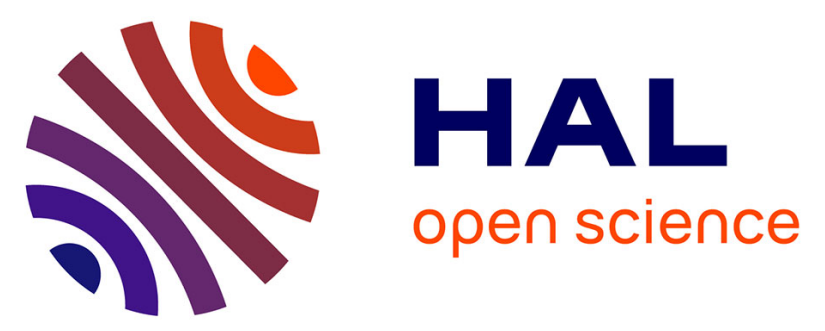

\title{
Multiple-Camera Instrumentation of a Single Point Incremental Forming Process Pilot for Shape and 3D Displacement Measurements: Methodology and Results
} Jean-José Orteu, Florian Bugarin, J. Harvent, Laurent Robert, Vincent Velay

\section{- To cite this version:}

Jean-José Orteu, Florian Bugarin, J. Harvent, Laurent Robert, Vincent Velay. Multiple-Camera Instrumentation of a Single Point Incremental Forming Process Pilot for Shape and 3D Displacement Measurements: Methodology and Results. Experimental Mechanics, 2011, 51 (4), pp.625-639. 10.1007/s11340-010-9436-1 . hal-01170378

\section{HAL Id: hal-01170378 \\ https://imt-mines-albi.hal.science/hal-01170378}

Submitted on 1 Jul 2015

HAL is a multi-disciplinary open access archive for the deposit and dissemination of scientific research documents, whether they are published or not. The documents may come from teaching and research institutions in France or abroad, or from public or private research centers.
L'archive ouverte pluridisciplinaire HAL, est destinée au dépôt et à la diffusion de documents scientifiques de niveau recherche, publiés ou non, émanant des établissements d'enseignement et de recherche français ou étrangers, des laboratoires publics ou privés. 


\title{
Multiple-Camera Instrumentation of a Single Point Incremental Forming Process Pilot for Shape and 3D Displacement Measurements: Methodology and Results
}

\author{
Jean-José Orteu* , Florian Bugarin, Jacques Harvent, \\ Laurent Robert, Vincent Velay \\ Université de Toulouse ; INSA, UPS, Mines Albi, ISAE ; ICA (Institut Clément \\ Ader) ; Campus Jarlard, F-81013 Albi, France \\ École des Mines Albi, Campus Jarlard, F-81013 Albi, France
}

\begin{abstract}
A multiple-camera system (more than two cameras) has been developed to measure the shape variations and the 3D displacement field of a sheet metal part during a Single Point Incremental Forming (SPIF) operation. The modeling of the multiplecamera system and the calibration procedure to determine its parameters are described. The sequence of images taken during the forming operation is processed using a multiple-view Digital Image Correlation (DIC) method and the 3D reconstruction of the part shape is obtained using a Sparse Bundle Adjustment (SBA) method. Two experiments that demonstrate the potentiality of the method are described.
\end{abstract}

Key words: Multiple cameras, Multiple-camera calibration, Digital Image Correlation (DIC), Sparse Bundle Adjustment (SBA), Shape Measurement, 3D Displacement Measurement, Single Point Incremental Forming (SPIF). 


\section{Introduction}

This paper presents a multiple-camera system (more than two cameras) that has been developed to measure the shape variations and the 3D displacement field of a sheet metal part during a Single Point Incremental Forming operation.

Single Point Incremental Forming (SPIF) is a sheet forming process that has gained interest recently in prototyping and small batch production. In this process, a sheet metal part (aluminum, steel) is formed in a stepwise fashion by a displacement-controlled hemispherical tool without the need for a supporting die [1] (see Figure 1).
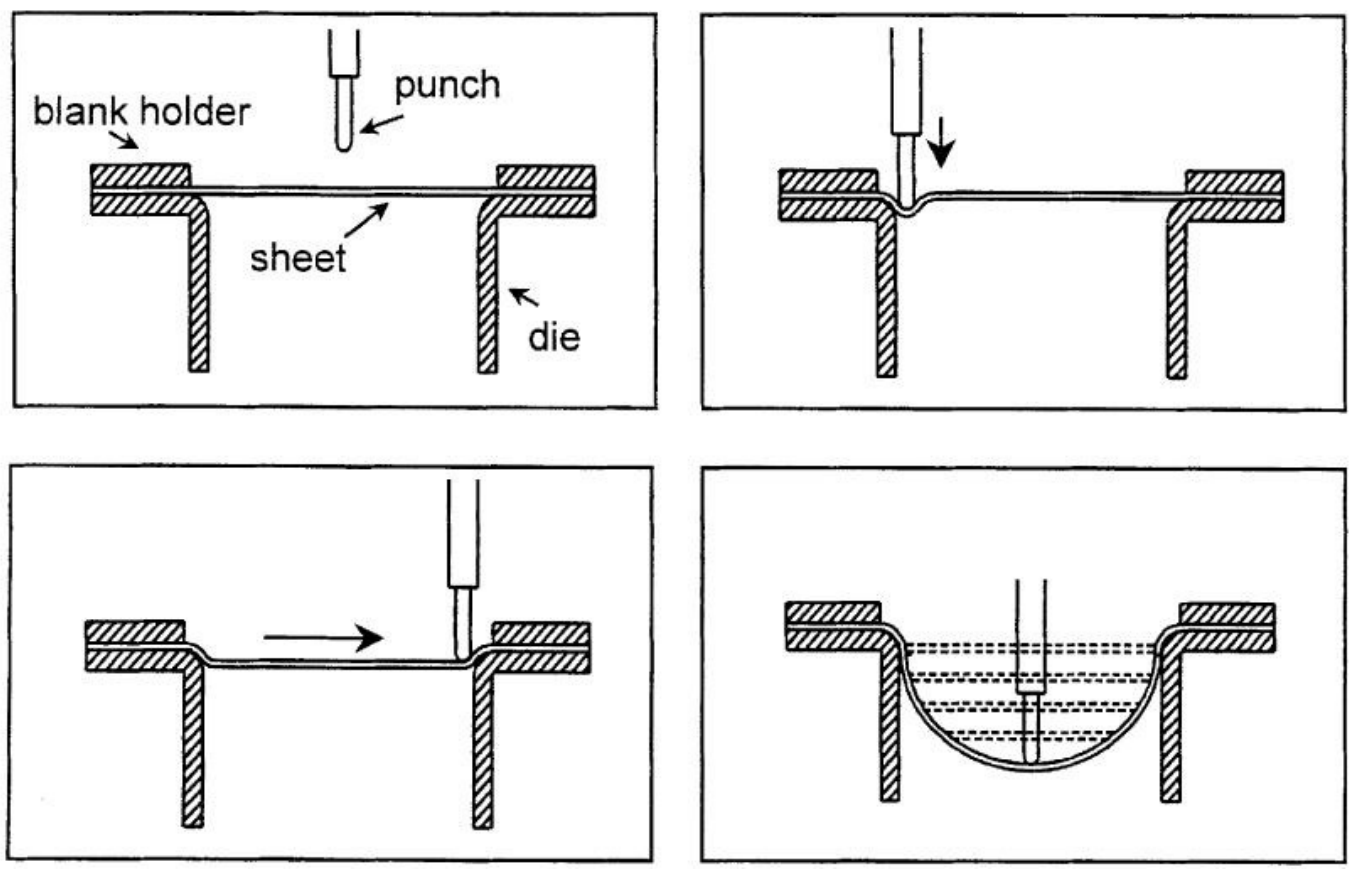

Fig. 1. The SPIF process: a sheet metal part is formed in a stepwise fashion by a displacement-controlled hemispherical tool without the need for a supporting die [5].

This process enables the manufacturing of a desired shape by an incremental deformation in a small contacted region. Because of this slicing technique, com-

* Corresponding author.

Email address: Jean-Jose.Orteu@mines-albi.fr (Jean-José Orteu). 
plicated products can be fabricated by using a simple shaped punch (driven by a numerically controlled milling machine or a robot) with a non-dedicated die. As a result it is characterized by a high flexibility which allows an interesting technology improvement to manufacture sheet metal parts in comparison with drawing process, and a reducing costs when prototypes or small batches have to be manufactured. Due to specific strain paths induced by the process and the fact that the plastic zone is strictly limited to the contact region between the tool and the work-piece, forming-limit curves and forming strategies are different from the classical deep drawing process $[2,3]$. It is reported however that accuracy of the obtained shape due to springback effect [4], heterogeneous thickness strain distribution [5] and time of the process are limitations of the process. Furthermore, simulations by Finite Element Methods are timeconsuming because of the incremental characteristics of this process [6]. However, these simulations need a good description of the process parameters, of the boundary conditions and of the material properties by the use of behavior model representative of the incremental characteristics of the process, taking into account, for instance, the history of strain paths during the process.

For studying these issues, a SPIF process pilot has been developed in our laboratory and instrumented with a force sensor to measure the resultant loading applied to the sheet metal part during the forming. It has been also instrumented with a multiple-camera system, which is described in detail in this paper, to measure the shape variation of the sheet metal part and the 3D displacement/strain field of its surface.

The outline of the paper is as follows: In Section 2, the SPIF process pilot and the multiple-camera system are first presented. In Section 3, the modeling of the multiple-camera system and the procedure developed to calibrate such a system are described. In Section 4, the multiple-image Digital Image Correlation (DIC) method that has been developed to solve the image matching problem is presented. In Section 5, the multiple-camera system is used to measure the 3D shape of a formed part (experiment I). In Section 6, 
the multiple-camera system is used to measure the 3D displacement field undergone by a sheet metal part during a Single Point Incremental Forming operation (experiment II).

\section{The SPIF Process Pilot and the Multiple-Camera System}

\subsection{The SPIF Process Pilot}

A SPIF process pilot (see Figure 2) has been developed in order to reproduce complex loading paths close to those induced in an industrial environment. Sheet deformation is performed with a $20 \mathrm{~mm}$ diameter hemispherical punch. A three axes motion of the tool $(X, Y, Z$ axis directions) is provided by three ball screw spindles. It allows on the one hand a vertical displacement and a local stamping of the sheet and, on the other hand, a horizontal displacement in the frame of the sheet leading to the incremental forming of the piece. A $400 \times 400 \mathrm{~mm}^{2}$ work area can be studied in the current configuration of the machine and a $100 \mathrm{~mm}$ depth of draw can be reached. In order to form the piece, a bottom-top forming direction was considered. As a consequence, in-situ deformation of the sheet can be easily observed and measured. Furthermore, a multi-axial sensor sets up the pilot that enables to monitor the three dimensional force measurement induced in the sheet by the punch displacement. The friction coefficients can be evaluated from this force measurement. The monitoring of the punch displacement and strength induced by the tool into the sheet is performed with a computer connected to the pilot.

\subsection{The Multiple-Camera System}

The SPIF process pilot has been equipped with a specially designed 4-camera system (see Figure 3). The four cameras (8 bit AVT Marlin F-145B2 cameras with a $1392 \times 1040$ pixels resolution equipped with $16 \mathrm{~mm}$ lenses) are connected 


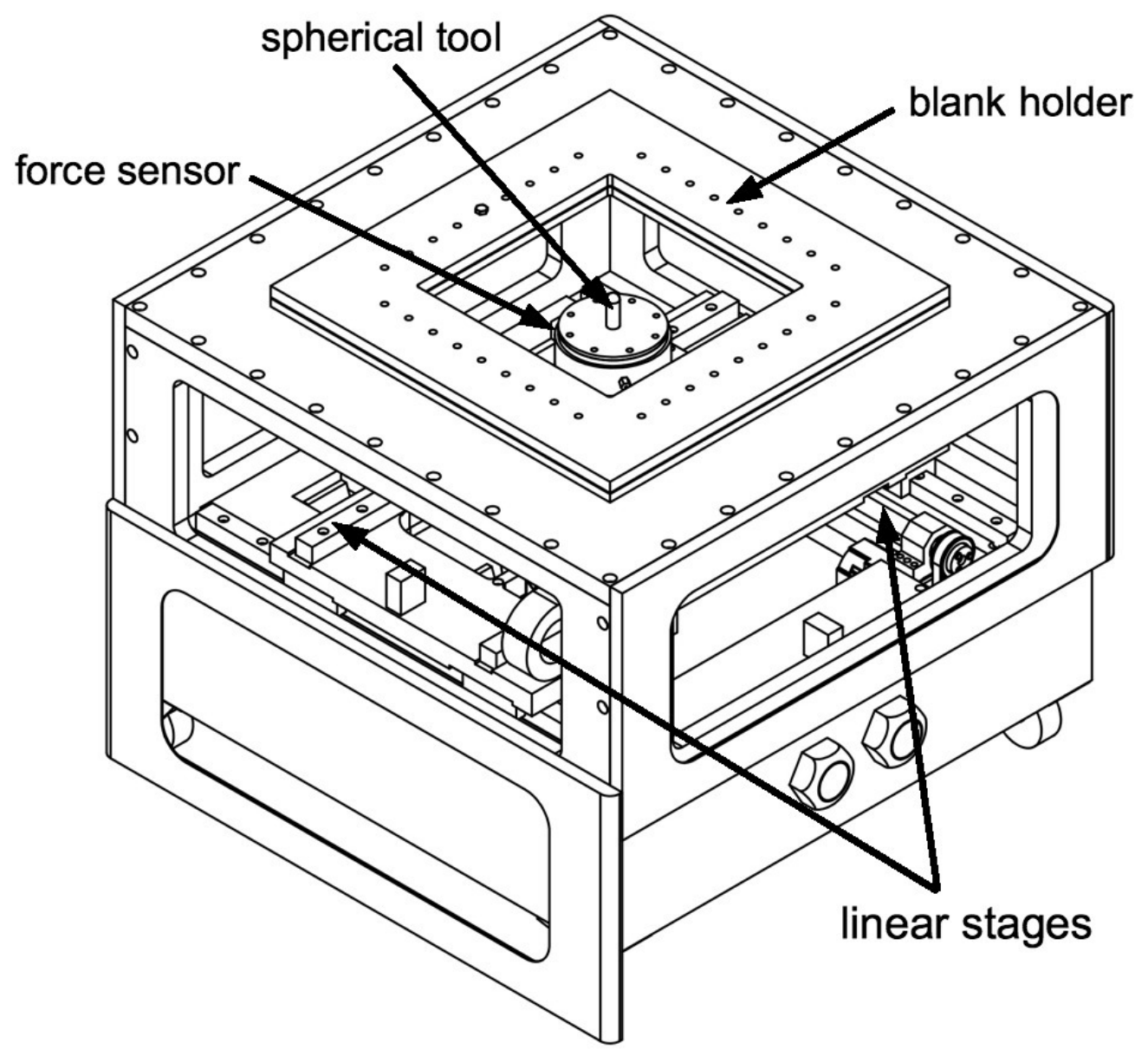

Fig. 2. The SPIF Process pilot.

to a PC through FireWire and the acquisition of four synchronized images is performed using the Vic-Snap ${ }^{\circledR}$ software [7].

Four cameras are used in order to be able to observe the part surface from different view points and thus to measure the 3D shape or the $3 \mathrm{D}$ displacement field of the whole part.

The measurements are obtained through three main steps:

(1) calibration of the N-camera system, prior to starting the forming (after this calibration step the $\mathrm{N}$-camera system is ready for measurement),

(2) acquisition of $\mathrm{N}$ synchronized images (i.e. one-shot image acquisition),

(3) processing of the $\mathrm{N}$ images in order to match corresponding points in these images (multiple-image matching). 


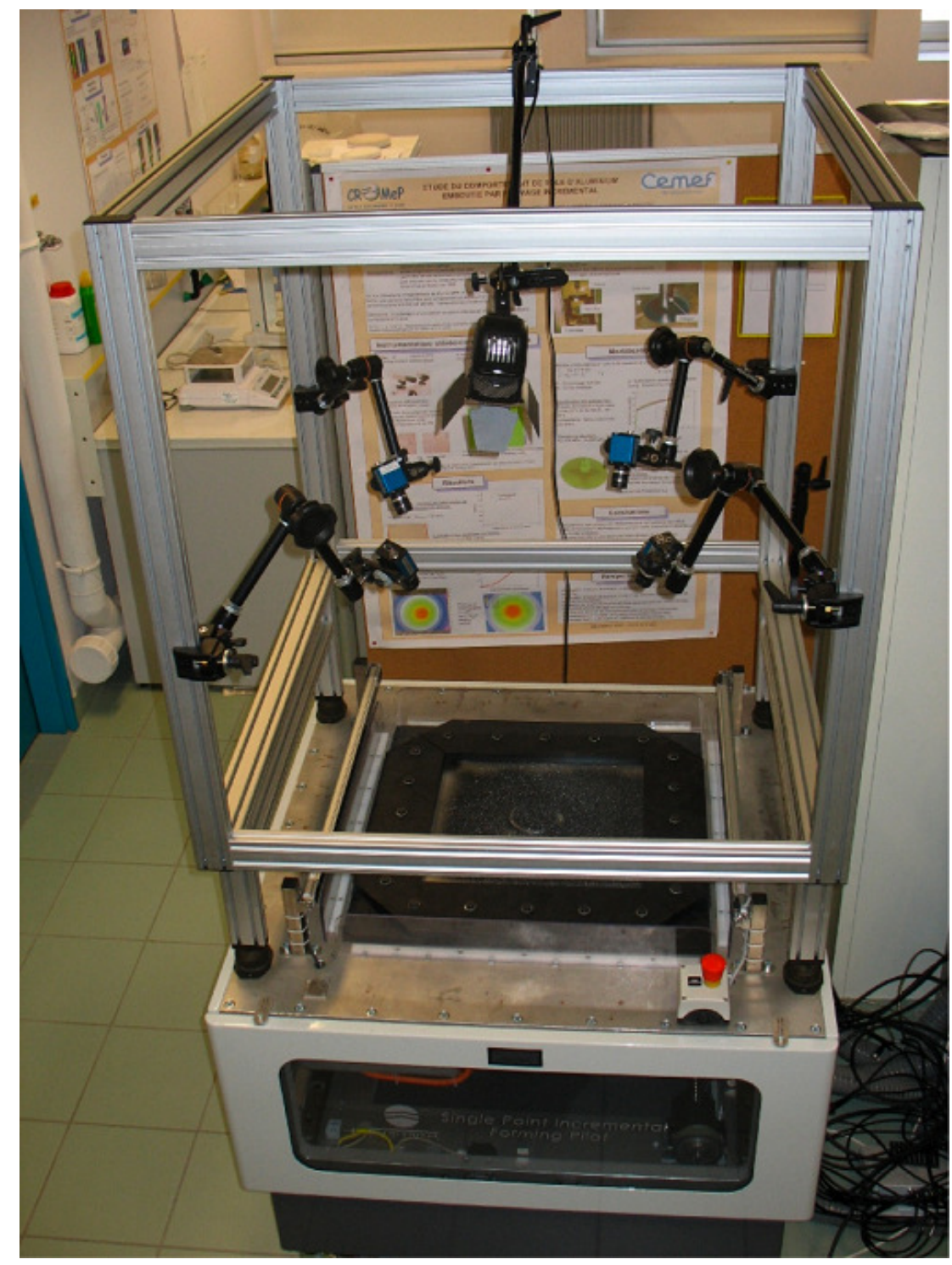

Fig. 3. The SPIF pilot instrumented with the 4-camera system.

Steps (2) and (3) can be repeated throughout the forming operation, leading (a) to the shape of the part at each acquisition time and (b) to the 3D displacement of its surface between each acquisition time. Note that in order to get the 3D displacement field of the part surface an image matching is also performed between images taken by the cameras at different acquisition time (temporal image matching described in Section 6).

In the present work, we have developed an innovative method to calibrate the $\mathrm{N}$-camera system as a whole, i.e. the $\mathrm{N}$ cameras are considered all together (see 
Section 3). Thanks to this approach, the whole 3D shape of the part or its 3D displacement field can be obtained directly, by a multiple-image triangulation method (see Section 5), from any set of image-points matched within all the images.

Note that in the present application, involving 4 cameras, an alternative approach could have been to consider the 4-camera system as two independent stereovision sensors (2 cameras plus 2 cameras, i.e. pairwise stereovision). In that case, each stereovision sensor can only measure a limited area of the part (the portion of the part observed by the stereovision sensor in its view field) and the 3D measurements provided by each stereovision sensor are independent. Thus, a so-called $3 D$ stitching operation is required in order to register in a common coordinate system the 3D measurements provided by each stereovision sensor. This 3D stitching operation, that relates the measurements provided by two independent sensors, can be automatized by using specific markers that are stuck onto the part surface before starting the forming. The $3 \mathrm{D}$ stitching operation can also be performed using DIC [9,11] (using for instance the "Multi-View" option in the Vic-3D ${ }^{\circledR}$ software [7]).

It is worth pointing out that our approach does not require any $3 \mathrm{D}$ stitching operation (unless we use several clusters of cameras in the case of very large objects as explained in Section 3.3), nor the use of markers, as the N-camera system $(N>2)$ provides directly all the $3 \mathrm{D}$ measurements in a common coordinate system.

It should be noted also that our approach can deal with an odd number of cameras. In that case, the stereovision approach mentioned above, which consists in segmenting the multiple-camera system into several clusters of two cameras (pairwise stereo), cannot be used.

The image matching is performed using the Digital Image Correlation (DIC) method [12]. The classical DIC method, used for instance in the 3D-DIC method for matching two stereo pairs of images, has been extended to perform multiple-view matching (see Section 4). 
It is well known that the DIC technique is efficient on textured objects. When an object does not present a suitable random, contrasted texture, one may apply a thin coating of high-contrast particles such as from spray painting [13], toner powder, ink, lithography, etc.

In the present work, prior to performing the experiment, a random black-andwhite speckle pattern was created at the surface of the sheet metal part by using spray paint.

Figure 4 shows the speckle-patterned sheet metal part being formed and the four images taken by the 4-camera system. It should be noted that for shape measurement no surface preparation is required as the speckle-pattern can be video-projected onto the part [8-10].

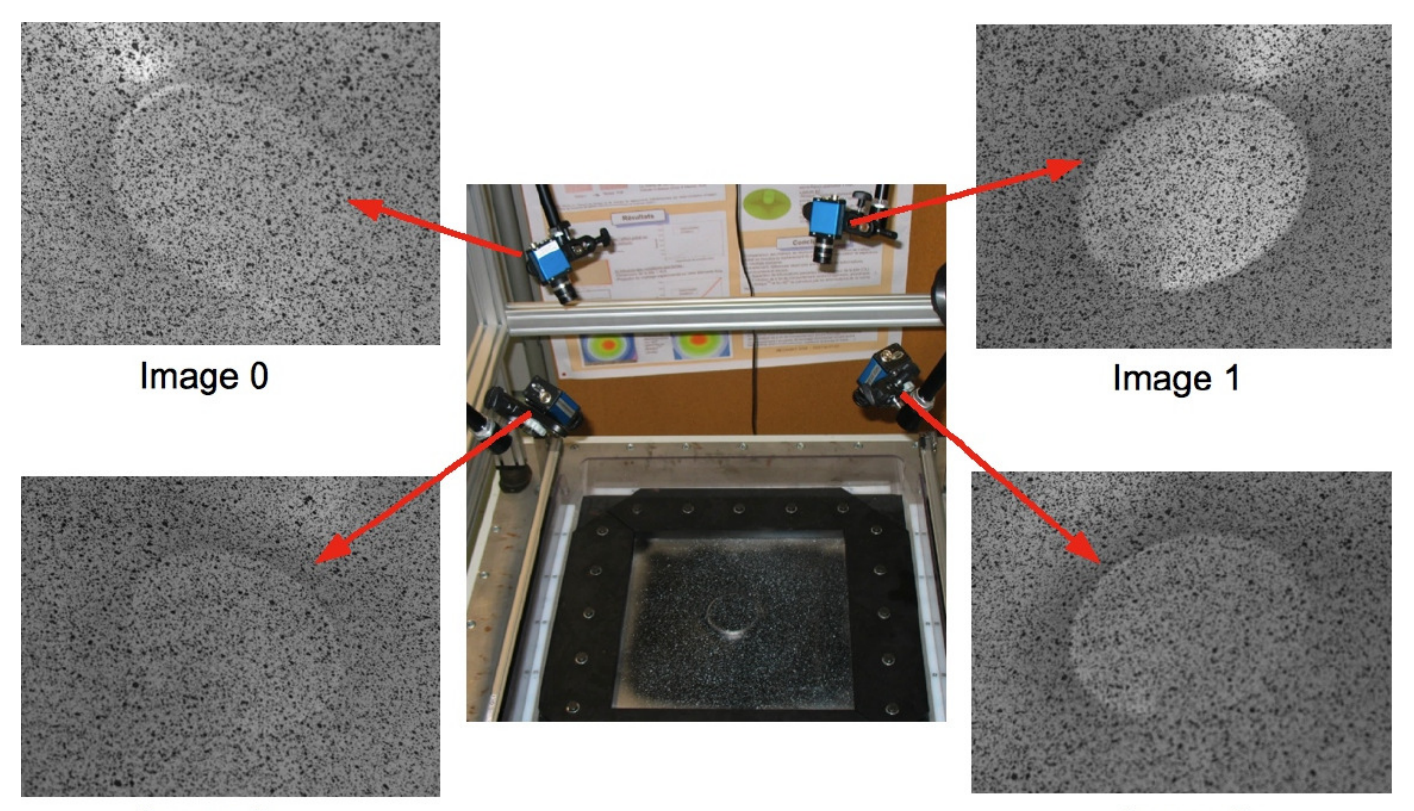

Image 2

Image 3

Fig. 4. The speckle-patterned sheet metal part and the four images taken by the 4-camera system.

Quite recently, Vasilakos et al. [14] have used the classical 3D-DIC technique (stereovision with only two cameras) to study the deformations of a two-anglepyramid sheet metal part during a Single Point Incremental Forming. Only a portion of the part has been investigated because two cameras were not enough to measure the whole part. 
Within an incremental forming robotized system, Sasso et al. [15] have proposed a fringe-projection based technique, using four cameras, to compute the whole shape of the formed part. Nevertheless, as the fringe-projection technique cannot give the surface displacement or strain field of the part, they had to use a complementary technique (the so-called "grid method") for measuring the deformation induced on the sheet metal part by the forming process.

Our multiple-camera-based measurement technique that will be described in the following sections provides directly the 3D shape or the 3D displacement field of the whole part.

\section{Modeling and Calibration of the Multiple-Camera System}

Camera calibration is an important task in 3D computer vision, particularly when metric data are required for applications involving accurate dimensional measurements.

The goal of camera calibration is to achieve the determination of two sets of parameters. The first set is called the extrinsic parameters and represents the position and orientation of each camera with respect to an arbitrary world reference frame. The second set is called intrinsic parameters and represents the internal parameters of each camera (like the focal length, the size of the pixels, the distortion parameters, etc.) $[16,17]$. These calibration data are required to compute, by triangulation, the $3 \mathrm{D}$ coordinates of a point corresponding to matched pixels in the images taken by at least two cameras (stereovision).

In the following sections, the modeling of the multiple-camera system and the optimization procedure used to compute the intrinsic and the extrinsic parameters are described. The modeling and the calibration of a single camera are presented in Appendix A in order to introduce the general methodology of our approach. 


\subsection{Modeling and Calibration of a Multiple-Camera System}

In previous work, the methodology described in Appendix A has been extended to the modeling and calibration of a stereovision sensor made up of two cameras $[18,19]$.

In the present work, we have extended this methodology to the modeling and calibration of a multiple-camera system made up of more than two cameras. Without any loss of generality, the methodology will be explained through the particular case of a 4-camera system (four cameras are used in the application described in the present paper).

\subsubsection{Modeling}

We consider that one camera is the master camera (camera \#0 in Figure 5).

The model will include four intrinsic parameters vectors (one vector for each camera), four distortion parameters vectors (one vector for each camera), and four rigid body transformations $\mathbf{L}, \mathbf{T}_{01}, \mathbf{T}_{02}, \mathbf{T}_{03} . \mathbf{L}=(\mathbf{R}, \mathbf{t})$ is the rigidbody transformation between the world coordinate system $\mathcal{R}_{\mathrm{w}}$ and the master camera coordinate system $\mathcal{R}_{\mathrm{c}}^{0}$ (see Figure 5).

The function that relates a $3 \mathrm{D}$ point in space $M$ and its four 2D projection $\left(m_{0}, m_{1}, m_{2}, m_{3}\right)$ into the four images can be written:

$$
\begin{aligned}
& f: \quad \mathbb{R}^{20} \times \mathbb{R}^{12} \times \mathbb{R}^{12} \times \mathbb{R}^{12} \times \mathbb{R}^{3} \quad \longmapsto \quad \mathbb{R}^{2} \times \mathbb{R}^{2} \times \mathbb{R}^{2} \times \mathbb{R}^{2} \\
& \left(\left(\begin{array}{c}
\mathbf{k}_{0} \\
\mathbf{k}_{1} \\
\mathbf{k}_{2} \\
\mathbf{k}_{3}
\end{array}\right),\left(\begin{array}{c}
\mathbf{d}_{0} \\
\mathbf{d}_{1} \\
\mathbf{d}_{2} \\
\mathbf{d}_{3}
\end{array}\right),\left(\begin{array}{c}
\mathbf{R} \\
\mathbf{R}_{01} \\
\mathbf{R}_{02} \\
\mathbf{R}_{03}
\end{array}\right),\left(\begin{array}{c}
\mathbf{t} \\
\mathbf{t}_{01} \\
\mathbf{t}_{02} \\
\mathbf{t}_{03}
\end{array}\right), M\right) \longmapsto\left(\begin{array}{c}
m_{0}=\mathrm{D}_{\mathbf{d}_{0}}\left(\mathbf{K}_{\mathbf{k}_{0}}(\mathbf{P}(\mathbf{L}(M)))\right), \\
m_{1}=\mathrm{D}_{\mathbf{d}_{1}}\left(\mathbf{K}_{\mathbf{k}_{1}}\left(\mathbf{P}\left(\mathbf{T}_{01}(\mathbf{L}(M))\right)\right)\right), \\
m_{2}=\mathrm{D}_{\mathbf{d}_{2}}\left(\mathbf{K}_{\mathbf{k}_{2}}\left(\mathbf{P}\left(\mathbf{T}_{02}(\mathbf{L}(M))\right)\right)\right), \\
m_{3}=\mathrm{D}_{\mathbf{d}_{3}}\left(\mathbf{K}_{\mathbf{k}_{3}}\left(\mathbf{P}\left(\mathbf{T}_{03}(\mathbf{L}(M))\right)\right)\right)
\end{array}\right)
\end{aligned}
$$




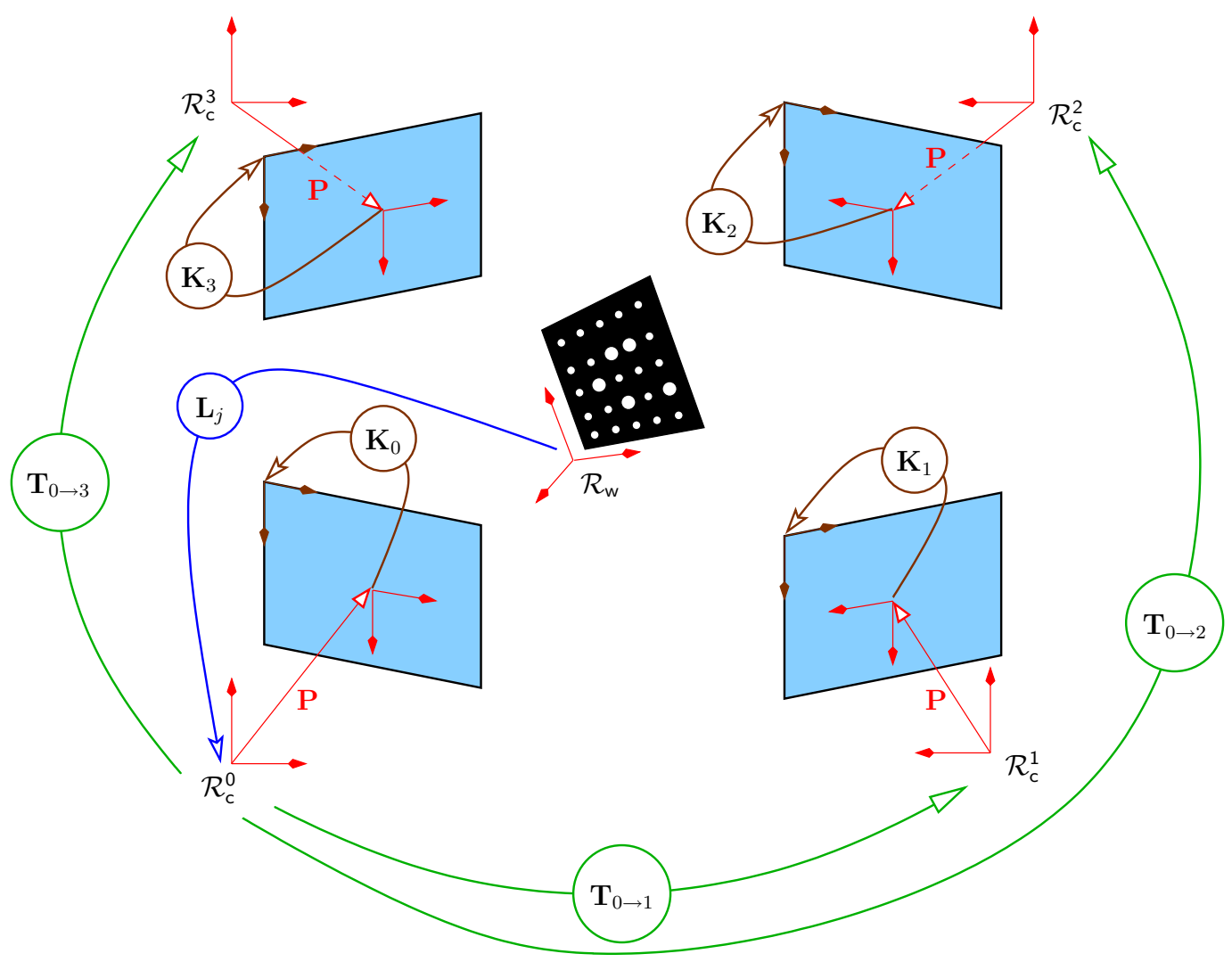

Fig. 5. Geometrical transformations involved in the modeling of the 4-camera system.

\subsubsection{Calibration}

The experimental procedure for determining the parameters of the multiplecamera system is derived from the procedure described in Appendix A and in Figure A.2 for the calibration of a single camera. The calibration is performed by acquiring a series of $n 4$-images (a total of $4 n$ images is acquired) of a planar calibration target made up of $p$ circular points, observed at different positions and orientations (four images are acquired at each position or orientation of the target). As in Appendix A, we define a set of $n$ localization matrices $\left(\mathbf{R}_{i}, \mathbf{t}_{i}\right)(i=1 \ldots n)$ that represent the $i^{\text {th }}$ position of the calibration target with respect to master camera reference frame.

By writing:

$$
\mathbf{k}=\left(\mathbf{k}_{0}, \mathbf{k}_{1}, \mathbf{k}_{2}, \mathbf{k}_{3}\right) \quad \mathbf{d}=\left(\mathbf{d}_{0}, \mathbf{d}_{1}, \mathbf{d}_{2}, \mathbf{d}_{3}\right)
$$




$$
\begin{array}{cl}
\mathbf{R}_{s}=\left(\mathbf{R}_{01}, \mathbf{R}_{02}, \mathbf{R}_{03}\right) & \mathbf{t}_{s}=\left(\mathbf{t}_{01}, \mathbf{t}_{02}, \mathbf{t}_{03}\right) \\
\mathbf{R}=\left(\mathbf{R}_{1} \ldots \mathbf{R}_{n}\right) & \mathbf{t}=\left(\mathbf{t}_{1} \ldots \mathbf{t}_{n}\right) \\
m_{i}^{j}=\left(m_{0, i}^{j}, m_{1, i}^{j}, m_{2, i}^{j}, m_{3, i}^{j}\right) & i=1 \ldots n ; j=1 \ldots p
\end{array}
$$

the calibration problem is solved by minimizing the following quantity:

$$
\underset{\mathbf{k}, \mathbf{d}, \mathbf{R}_{s}, \mathbf{t}_{s}, \mathbf{R}_{i}, \mathbf{t}_{i}, M}{\operatorname{Min}} \sum_{i=1}^{n} \sum_{j=1}^{p}\left\|m_{i}^{j}-f\left(\mathbf{k}, \mathbf{d}, \mathbf{R}_{s}, \mathbf{t}_{s}, \mathbf{R}_{i}, \mathbf{t}_{i}, M_{j}\right)\right\|^{2}
$$

This equation can be written in the form:

$$
\underset{\mathbf{k}, \mathbf{d}, \mathbf{R}_{s}, \mathbf{t}_{s}, \mathbf{R}_{i}, \mathbf{t}_{i}, M}{\operatorname{Min}} \sum_{i=1}^{n} \sum_{j=1}^{p} \sum_{k=0}^{3} d_{i j k}^{2}
$$

In Eq. (3), $d_{i j k}$ represents, for camera number $k$, the 2D Euclidean distance in the image (in pixel units) between the $2 \mathrm{D}$ features $m_{i}^{j}$ extracted in the image of the target and the projection, through the camera model, of the 3D corresponding points $M_{j}$.

There are $32+18+6 n+3 p$ parameters to be estimated ${ }^{1}$ and $4 \times 2 n p$ equations $^{2}$.

A judicious choice of $n$ and $p$ leads to an over-determined system (more equations than unknowns). The minimization of Eq. (2) is solved using a Sparse

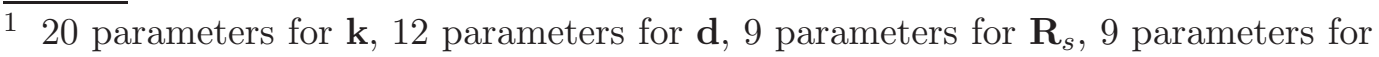
$\mathbf{t}_{s}, 3$ parameters for each $\mathbf{R}_{i}, 3$ parameters for each $\mathbf{t}_{i}$ and 3 parameters for each $3 \mathrm{D}$ point $M$.

$24 \times 2 n p$ is a maximum, corresponding to the situation where all the $p$ points of the calibration target are seen by all the 4 cameras for all the $n$ positions of the target. In practice, the number of equations $N_{e}$ is a little smaller since in Eq. (3) the $d_{i j k}$ terms are only computed for the image-points that are seen in at least two cameras. 
Bundle Adjustment (SBA) method based on the Levenberg-Marquardt algorithm [20]. In order the algorithm to converge, an initial guess of the parameters is required. This initial guess is obtained using two successive steps:

(1) the parameters of each camera are estimated by minimizing Eq. (A.3) (see Appendix A).

(2) the parameters of 3 stereo systems ([camera \#0 + camera \#1], [camera $\# 0+$ camera \#2], [camera \#0 + camera \#3]) are estimated in order to get an initial guess for $\mathbf{R}_{s}, \mathbf{t}_{s}, \mathbf{R}$ and $\mathbf{t}$.

The quality of the calibration can be estimated using the global reprojection error, which is the residual obtained after minimizing Eq. (2).

In practice the quality of the calibration is assessed by computing the mean, the standard deviation and the RMS of the individual reprojection errors $d_{i j k}$ (see Eq. (3)), after the minimization of Eq. (2) is completed.

Thus the following quantities are calculated:

$$
\begin{aligned}
\bar{d} & =\frac{1}{N_{e}} \sum_{k}^{N_{e}} d_{k} \\
\sigma_{d} & =\sqrt{\frac{1}{N_{e}} \sum_{k}^{N_{e}}\left(d_{k}-\bar{d}\right)^{2}} \\
\mathrm{RMS}_{d} & =\sqrt{\frac{1}{N_{e}} \sum_{k}^{N_{e}} d_{k}^{2}}
\end{aligned}
$$

where $N_{e}$ is the number of equations, i.e. twice the number of $d_{i j k}$ terms taken into account in Eq. (3).

It should be noted that $\sum_{k}^{N_{e}} d_{k}^{2}$ is the residual of Eq. (2).

Another criterion is used to estimate the quality of the calibration: the computation of the mean epipolar distance between a set of image-points and their corresponding epipolar lines $[16,21]$. 


\subsection{Calibration results}

In the two experiments reported in Section 5 (experiment I) and Section 6 (experiment II), the multiple-camera system shown in Figures 3 and 4 has been calibrated using the methodology described in Section 3.1. The configuration of the cameras was different for each experiment.

In both experiments, the calibration target with $p=81$ circular dots has been moved in the field of view of the cameras.

In experiment I, $n=19$ positions of the target have been used ${ }^{3}$, leading to 395 parameters to be estimated ${ }^{4}$ and $N_{e}=12196$ equations.

In experiment II, $n=23$ positions of the target have been used leading to 419 parameters to be estimated and $N_{e}=14904$ equations.

Tables 1 to 4 give the calibration results for these two experiments along with the quality evaluation using the "reprojection error" criterion and the "mean distance to the epipolar lines" criterion.

Tables 2 and 4 show that an accurate calibration can be achieved with the proposed method (RMS of 0.077 pixel for experiment I and RMS of 0.032 pixel for experiment II).

It is noted that the calibration for experiment I was not as good as the calibration for experiment II. In experiment I (3D shape measurement of a pyramidal part), we wanted to test the limits of our method (in terms of calibration and

$\overline{3}$ Typically, from 15 to 25 images of the target are taken during the calibration step. The estimation of the calibration parameters is performed by using the "best" target positions leading to the best reprojection error (19 target positions in the experiment under concern).

${ }^{4}$ In both experiments, the skew factor is set to 0 and we only estimate the 1st order radial distortion parameter. Thus, there are only 20 intrinsic parameters: 16 parameters for $\mathbf{k}$ and 4 parameters for $\mathbf{d}$. 


\begin{tabular}{|c|c|c|c|c|c|}
\hline & $c_{x}$ & $c_{y}$ & $f_{x}$ & $f_{y}$ & $d_{1}$ \\
$($ pixel) & $($ pixel) & $($ pixel) & $($ pixel) & \\
\hline camera 0 & 696.2 & 507.8 & 3611.3 & 3610.3 & -0.356 \\
\hline camera 1 & 691.1 & 485.6 & 3606.1 & 3606.5 & -0.351 \\
\hline camera 2 & 672.0 & 527.1 & 3605.4 & 3605.4 & -0.340 \\
\hline camera 3 & 700.3 & 491.3 & 3592.3 & 3592.5 & -0.355 \\
\hline
\end{tabular}

Table 1

Calibration of the multiple-camera system: intrinsic parameters of experiment I (1st radial distortion model with skew factor set to 0 ).

\begin{tabular}{|c|c|c|c|c|c|}
\hline \multicolumn{4}{|c|}{ reprojection error } & \multicolumn{2}{c|}{ mean distance to epipolar lines } \\
\hline$N_{e}$ & $\bar{d}$ & $\sigma_{d}$ & $\mathrm{RMS}_{d}$ & $\#$ of points & mean distance \\
& $($ pixel $)$ & $($ pixel $)$ & $($ pixel $)$ & & (pixel) \\
\hline 12196 & 0.021 & 0.071 & 0.077 & 1517 & 0.49 \\
\hline
\end{tabular}

Table 2

Calibration quality of experiment I.

DIC-based image matching) and we put the cameras in such a position that the occultation problem was amplified (see images on Figure 8). Even in such a disadvantageous situation the calibration of the 4-camera system has been possible, with a calibration quality not so excellent.

\subsection{Discussion on multiple cameras issues}

From a general point of view, two main configurations of a multiple-camera system can be considered, depending on the application:

- a "surrounding cameras" configuration where several cameras are placed all 


\begin{tabular}{|c|c|c|c|c|c|}
\hline & $c_{x}$ & $c_{y}$ & $f_{x}$ & $f_{y}$ & $d_{1}$ \\
$($ pixel) & $($ pixel) & $($ pixel) & $($ pixel) & \\
\hline camera 0 & 698.7 & 503.3 & 3601.6 & 3601.0 & -0.352 \\
\hline camera 1 & 705.9 & 526.1 & 3592.8 & 3593.0 & -0.338 \\
\hline camera 2 & 696.6 & 508.0 & 3584.6 & 3584.6 & -0.345 \\
\hline camera 3 & 685.4 & 480.6 & 3616.6 & 3617.0 & -0.352 \\
\hline
\end{tabular}

Table 3

Calibration of the multiple-camera system: intrinsic parameters of experiment II (1st radial distortion model with skew factor set to 0 ).

\begin{tabular}{|c|c|c|c|c|c|}
\hline \multicolumn{4}{|c|}{ reprojection error } & \multicolumn{2}{c|}{ mean distance to epipolar lines } \\
\hline$N_{e}$ & $\bar{d}$ & $\sigma_{d}$ & $\mathrm{RMS}_{d}$ & $\#$ of points & mean distance \\
& $($ pixel) & $($ pixel) & $($ pixel) & & (pixel) \\
\hline 14904 & 0.011 & 0.029 & 0.032 & 1863 & 0.19 \\
\hline
\end{tabular}

Table 4

Calibration quality of experiment II.

around an object. This kind of configuration is useful to get a one-shot 3D shape of the whole object. This configuration has been used in the work reported in the present paper.

- a "wall-of-cameras" configuration where several cameras are distributed over a quasi-planar structure facing the object to be measured. This kind of configuration is useful when a very large field of view is required to measure one face of a very large object (surface of several square meters). This kind of configuration has been used in our work reported in [8-10].

Of course, for large objects which complete shape is required in one-shot a combination of both configurations can be imagined, where the object is surrounded by several cameras or walls of cameras. 
The "wall-of-cameras" configuration is well-adapted for large volume applications requiring a large number of cameras, which fields of view do not necessary overlap. In that case, the multiple-camera system needs to be segmented into several clusters of cameras (from 2 to 5 cameras per cluster). Each cluster is calibrated using our multiple-camera system calibration method and the clusters are stitched together. In [8-10] for instance, we have successfully used a 8-camera system to perform the NDT inspection of large aeronautical structures. Two clusters of four cameras have been calibrated and the two clusters have been stitched together [10].

Regarding the size of the clusters, our bundle-adjustment-based method can theoritically be used to calibrate a great number of cameras. For instance, with simulated data we have been able to calibrate up to 10 cameras. In practice, in our applications, we have calibrated up to 5 cameras as a whole. The limiting factor is the practical methodology that we use to calibrate the cameras: a calibration target is moved in the view-field of the cameras and the proposed multiple-camera model requires that all the calibration views be visible by at least one camera (the so-called "master camera"). This is not really a constraint when the cameras are placed in the "surrounding cameras" configuration with a master camera having an overlapping field of view with all the other cameras. The proposed practical methodology is more difficult to use when the "wall-of-cameras" configuration is required, as the fields of view do not overlap necessary. In that case, the set of cameras cannot be calibrated as a whole and needs to be segmented into smaller clusters. We are currently thinking about a new multiple-camera model that will overcome this limitation.

\section{Multiple-image Digital Image Correlation}

Once the multiple-camera system has been calibrated its geometry is known and the $3 \mathrm{D}$ reconstruction of a point in space can be obtained by multiple- 
image triangulation [17] as shown in Figure 6.

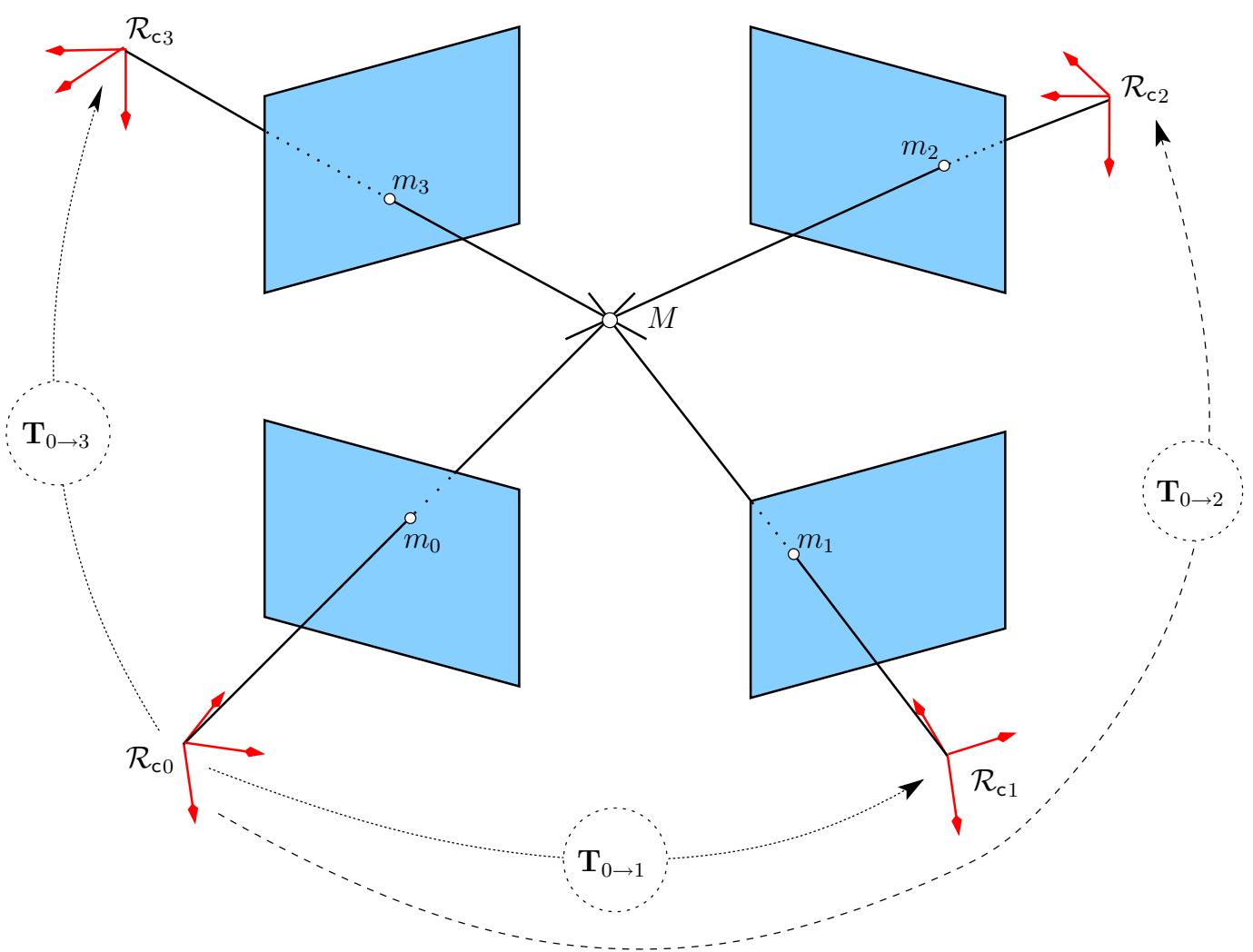

Fig. 6. Multiple-image triangulation. $M$ can be reconstructed in 3D from the four matched image-points $\left(m_{0}, m_{1}, m_{2}, m_{3}\right)$ and from the three transformations $\mathbf{T}_{0 \rightarrow 1}$, $\mathbf{T}_{0 \rightarrow 2}, \mathbf{T}_{0 \rightarrow 3}$.

As shown in Figure 6 the triangulation process requires that the image-points be matched in all the images. Thus, a multiple-images Digital Image Correlation method has been developed to solve this image matching problem.

The DIC method is widely used in the computer vision community to establish pixel correspondences between images. Based on our previous work on 3DDIC (also called stereo-correlation) [12, 19,22-24], the DIC method has been extended in the current work to multiple-images matching. As mentioned in Section 2.2, prior to performing the experiment, a random black-and-white speckle pattern was created at the surface of the sheet metal part by using spray paint, in order to get a suitable, contrasted texture for DIC. 
In the (simple/theoretical) situation when all the points to be reconstructed are visible in one of the images (no problem of occultation), this image is taken as the reference image $\left(I_{0}\right)$ and a set of image-points (distributed over a virtual mesh ${ }^{5}$ ) are chosen in this image (see Figure 7). All these image-points are matched by DIC in the three other images $\left(I_{1}, I_{2}\right.$ and $\left.I_{3}\right)$, leading to a set of 4-matched pixels $\left(m_{0}, m_{1}, m_{2}, m_{3}\right)$.

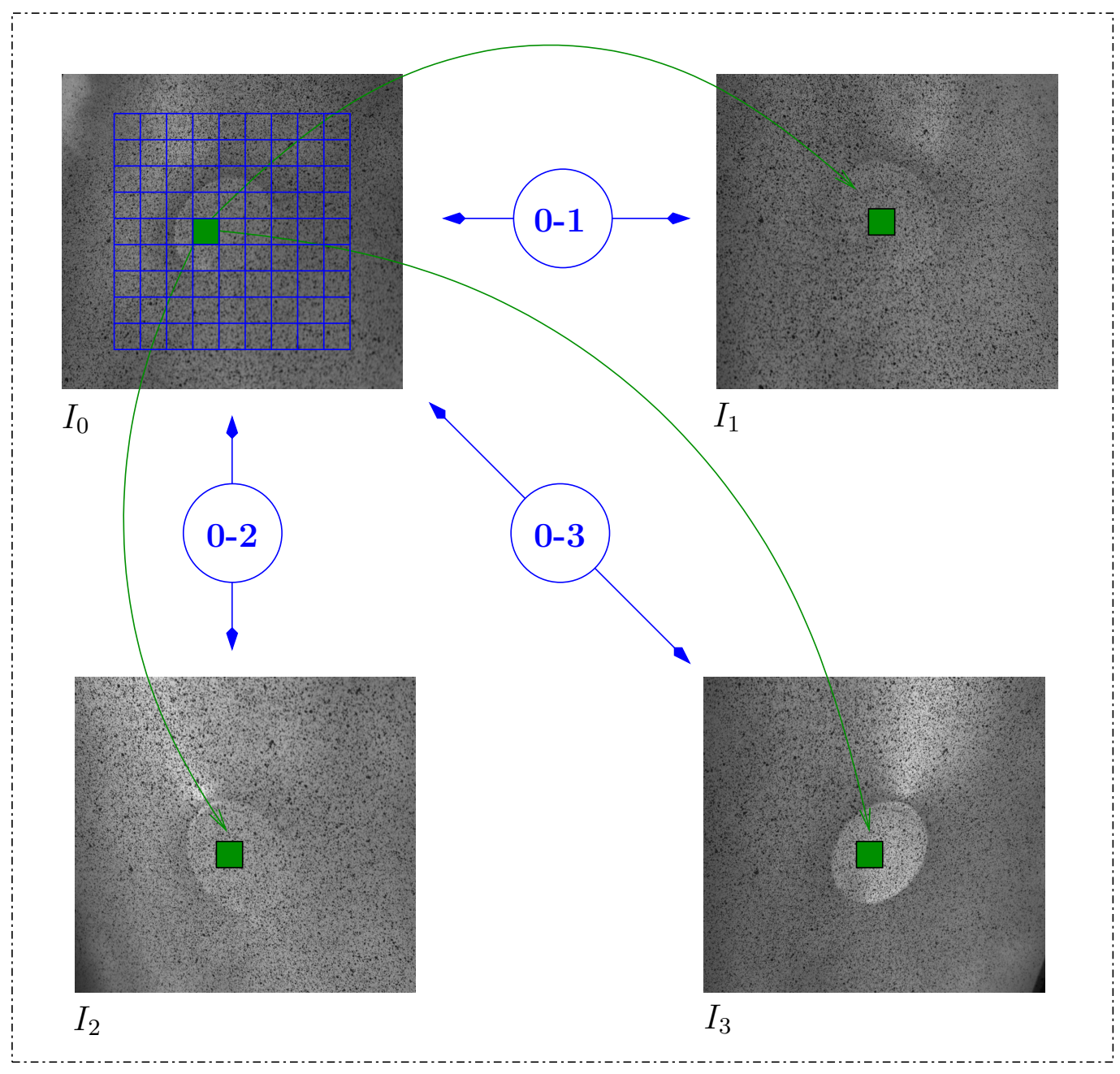

Fig. 7. Multiple-image DIC method for matching a set of 4 synchronized images taken during the forming of the part: the top left image is taken as the reference image and all the grid image-points are matched by DIC in the three other images.

A more realistic situation is the one presented in Figure 8. As can be seen 5 The mesh size is smaller than the one shown in Figure 7. Typically, the grid size is $5 \times 5$ pixels. 
on this figure, due to occultation problems, some points are visible on some images but are not visible on the others, which requires a careful handling of the image set. Indeed, in this situation, the set of matched points provided by the DIC process will depend on which image is taken as the reference image and some points will not be matched if they are not visible in the chosen reference image. The solution we have implemented consists in taking each image as the reference image successively and in performing several DIC operations $\left(I_{0} \rightarrow I_{1}, I_{0} \rightarrow I_{2}, I_{0} \rightarrow I_{3}, I_{1} \rightarrow I_{0}, I_{1} \rightarrow I_{2}, I_{1} \rightarrow I_{3}\right.$, etc.). In this way, all the points of the part surface visible in at least two cameras will be reconstructed in $3 \mathrm{D}$ during the $3 \mathrm{D}$ shape reconstruction phase.

\section{3D Shape Measurement by Multiple-image Triangulation and Bundle Adjustment}

\subsection{Methodology}

Using the calibration parameters of the multiple-camera system a multipleimages triangulation method (see Figure 6) has been implemented to compute the $3 \mathrm{D}$ position of all the matched image-points provided by the multipleimage DIC process. The 3D reconstruction process is completed using a Bundle Adjustment method [25].

It should be noted that before performing the triangulation, all the matched image-points have been corrected from their distortion beforehand using the distortion parameters provided by the camera calibration procedure. It has been shown in previous work $[19,26]$ that removing the distortion from the images leads to more accurate 3D measurements. 


\subsection{Results (experiment I)}

Our multiple-image-based 3D shape measurement method has been applied for measuring the shape of a formed part with a pyramidal shape (experiment I).

First, the 4-camera system has been calibrated using the method described in Section 3. The calibration results are reported in Tables 1 and 2.

Figure 8 shows four images of the formed part taken by our 4-camera system.
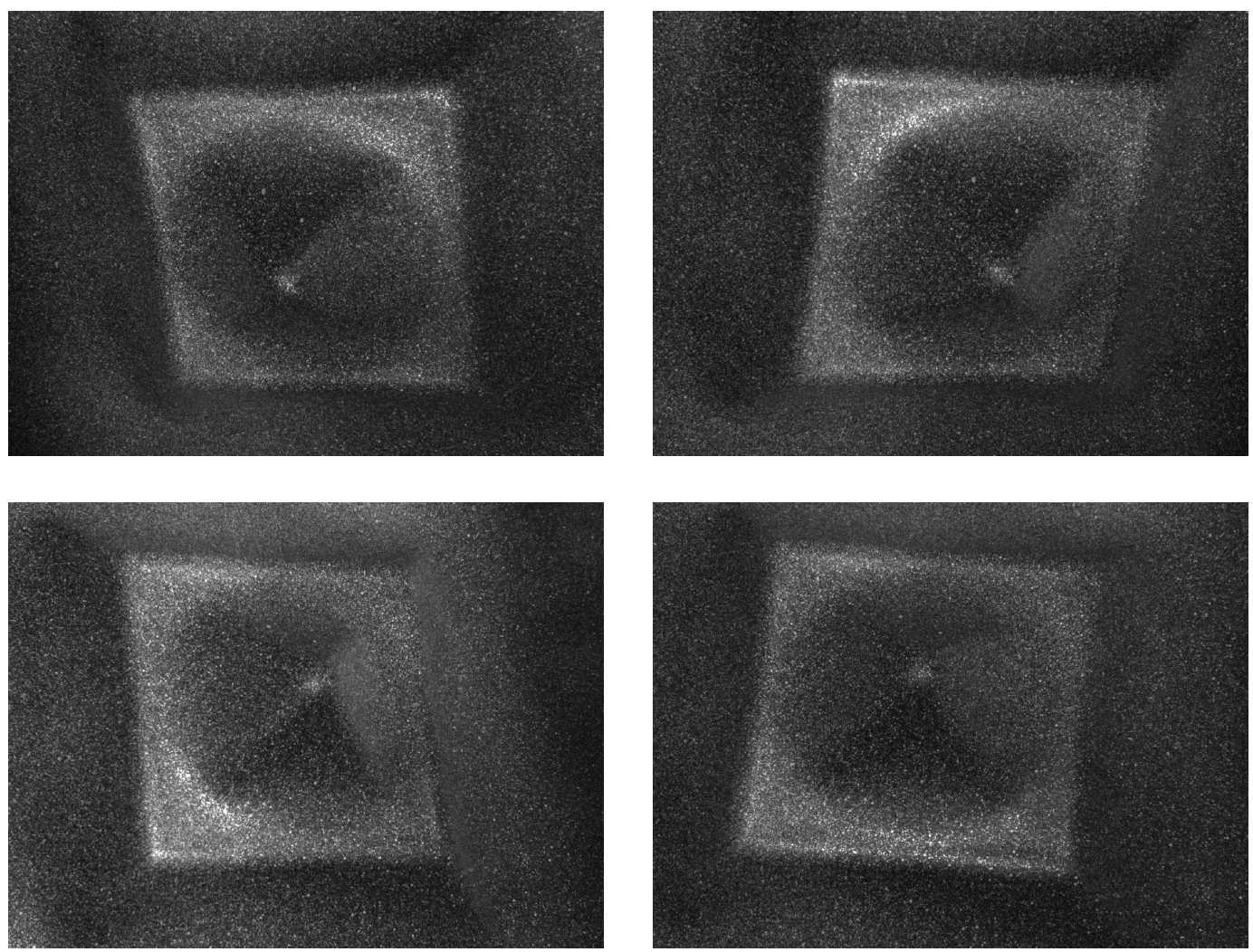

Fig. 8. Four images of a formed part with a pyramidal shape taken by the 4-camera system.

Figure 9 shows the 3D shape of the part computed by our method using the four images shown in Figure 8. 


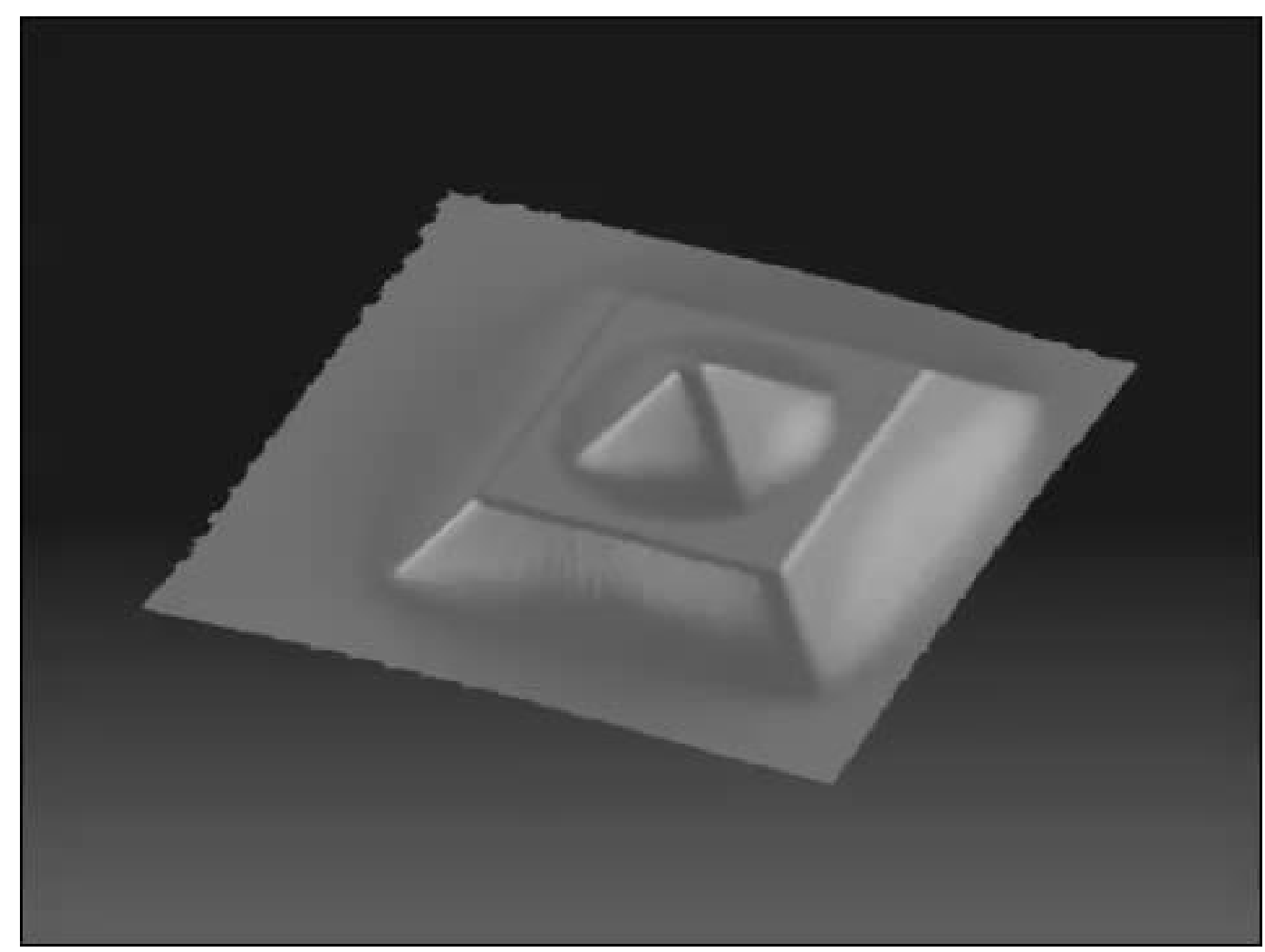

Fig. 9. The 3D shape of the formed pyramidal part computed by our method using the four images shown in Figure 8.

\subsection{Evaluation and discussion}

For comparison, the formed part has been also measured using the commercial HandyScan ${ }^{\circledR}$ hand-held laser scanner system. This scanning system needs to be pointed towards the object to be scanned and moved around the object. At each sensor position a partial shape reconstruction is performed. The dot markers stuck on the part surface that can be seen in Figure 10 have been specially added for HandyScan ${ }^{\circledR}$ measurements (they are not used for measuring the part with our DIC-based method). They are used by the laser scanner system in order to register in a common coordinate system the 3D measurements computed from each sensor position.

The shape computed by our multiple-camera DIC-based method and the one obtained by the HandyScan ${ }^{\circledR}$ system have been compared using the 


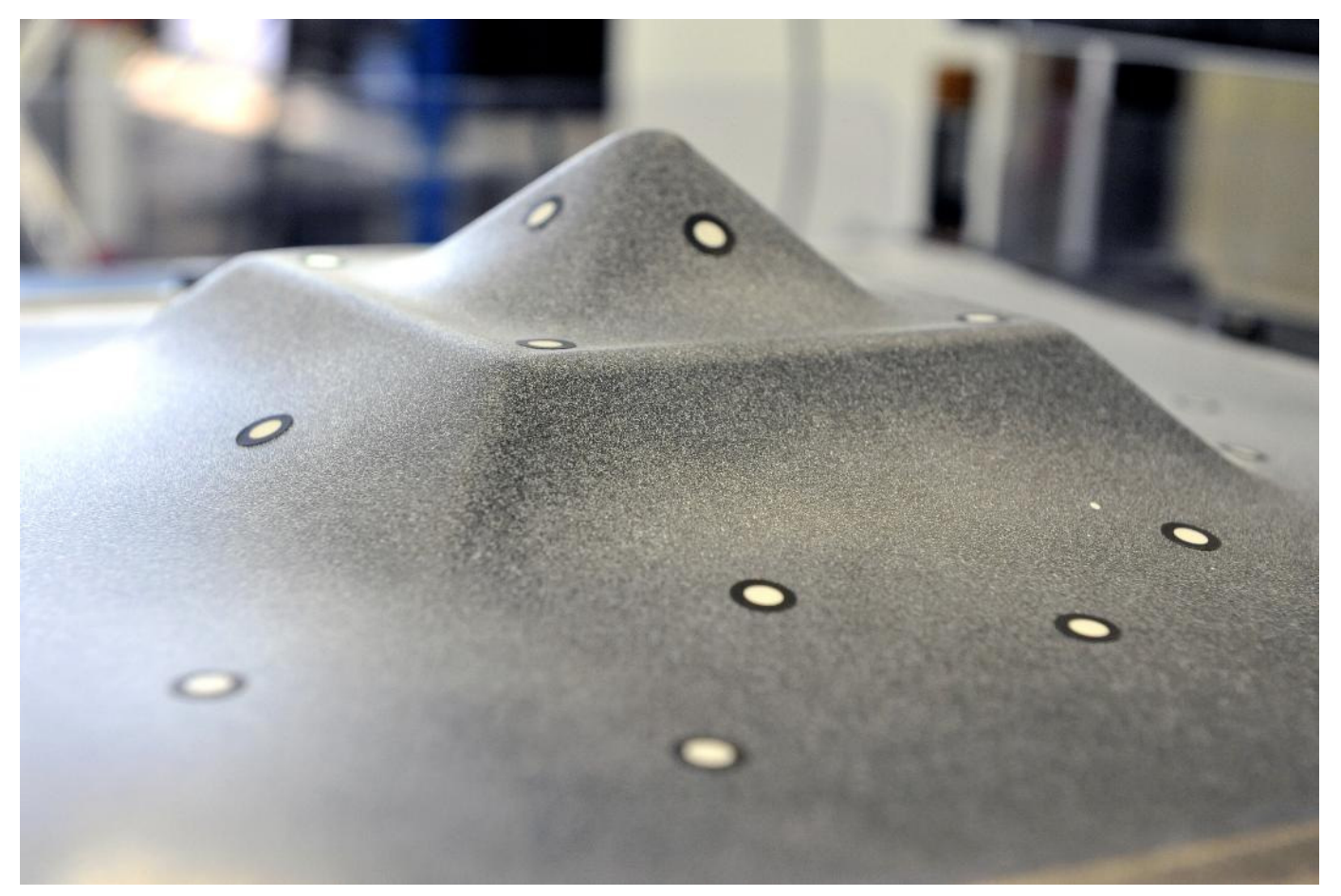

Fig. 10. The part with the fiducial markers put onto it to perform the $3 \mathrm{D}$ shape measurement with the HandyScan ${ }^{\circledR}$ hand-held laser scanner.

Geomagic ${ }^{\circledR}$ Qualify ${ }^{\circledR}$ software (see Figure 11).

Figure 11 shows that the mean difference between the shape computed by our multiple-camera DIC-based method and by the HandyScan ${ }^{\circledR}$ system is about $0.05 \mathrm{~mm}$, which is a very good result, given the fact that the HandyScan ${ }^{\circledR}$ accuracy announced by the manufacturer is of the same order.

Moreover, as it can be seen on that figure, the mean difference is contaminated by some badly measured areas (blue areas, for which the shape deviation is much higher than $0.05 \mathrm{~mm}$ ). Figure 11 clearly shows that some of these 


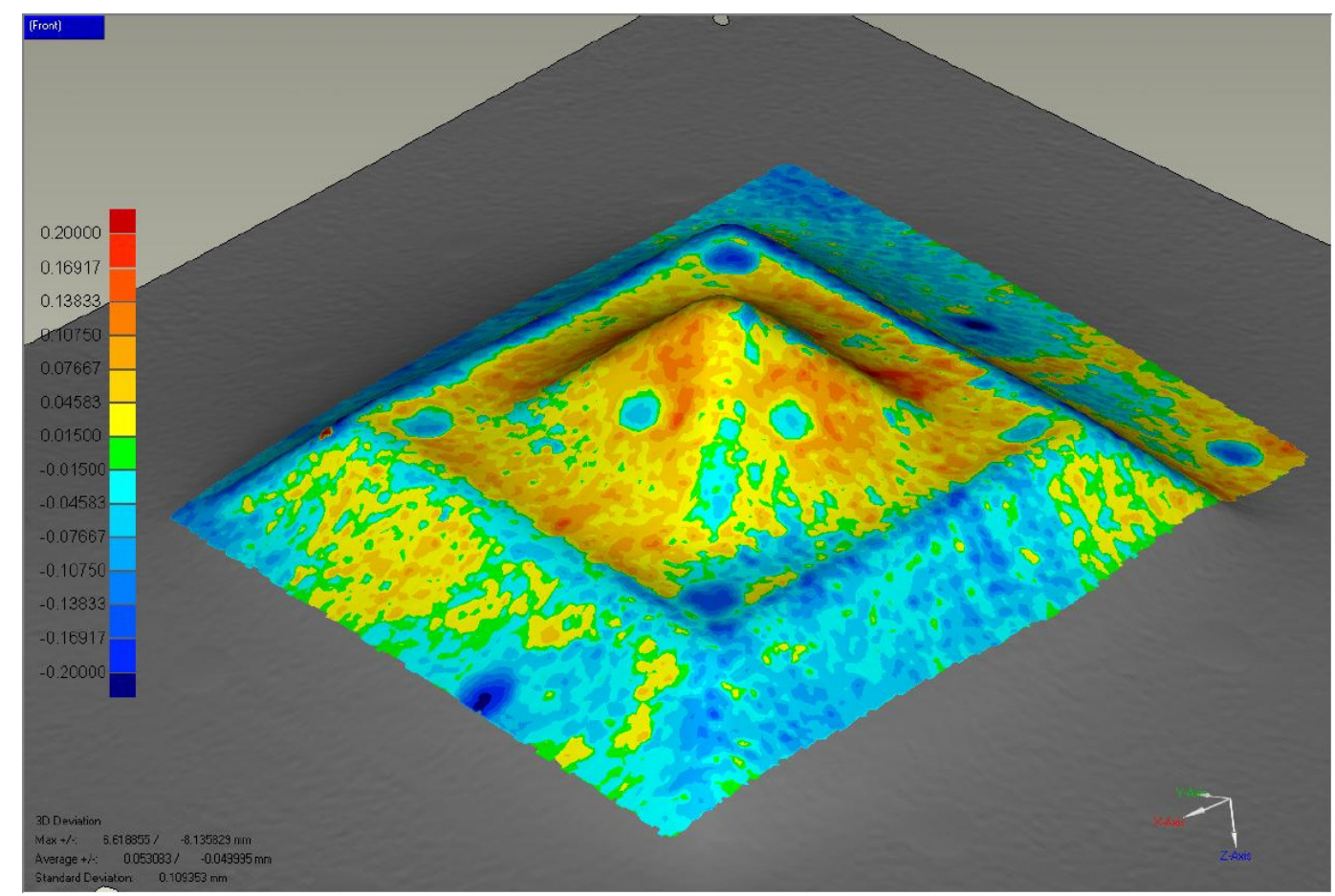

Fig. 11. The two shapes computed by our multiple-camera DIC-based method and by the HandyScan ${ }^{\circledR}$ system have been compared using the Geomagic ${ }^{\circledR}$ Qualify ${ }^{\circledR}$ software.

areas correspond to the circular markers that have been stuck on the part surface for the HandyScan ${ }^{\circledR}$ measurement after measuring the part with our multiple-camera DIC-based method. All these statements show that a good measurement accuracy (better than $0.05 \mathrm{~mm}$ on a $400 \times 400 \times 100 \mathrm{~mm}^{3}$ part) can be achieved by the proposed method.

Finally, note that the HandyScan ${ }^{\circledR}$ system cannot perform a one-shot measurement of the whole part shape, whereas our 4-camera system can do it, by 
processing the four synchronized images taken simultaneously.

\section{3D Displacement Measurement by combining spatial and tem- poral DIC-based image matching}

\subsection{Methodology}

The methodology described in Sections 4 and 5 applied to a first set of four images taken by the 4 -camera system at time $t_{0}$ leads to the $3 \mathrm{D}$ shape of the part at this time. During the incremental forming process, the part is undergoing some deformations and its shape varies over time. A second set of four images is taken by the 4 -camera system at time $t_{1}$. The $3 \mathrm{D}$ displacement field of the part between time $t_{0}$ and time $t_{1}$ can then be computed by processing the two sets of four synchronized images taken at both instants (eight images to be processed).

This methodology of multiple-image matching can be repeated throughout the forming operation, leading (a) to the shape of the part at each acquisition time and (b) to the 3D displacement field of its surface between each acquisition time.

The computation method (derived from what we developed in [22] in the case of stereovision measurements involving only two cameras) is summarized in Figure 12. This figure shows how the multiple-image DIC-based matching is performed throughout the sequence of 4-images. As it can be seen in this figure, DIC is used for matching images taken at the same instant (spatial matching) but also for matching images taken at two different instants (temporal matching).

Let's consider that the sequence of 4-images has been processed between time $t_{0}$ and time $t_{0}+\Delta_{t-1}$. Figure 12 shows that the matching between time $t_{0}$ 
and time $t_{0}+\Delta_{t}{ }^{6}$ utilizes the image-points matched at time $t_{0}+\Delta_{t-1}$ as initial guesses. The subset shape function parameters of these matches are also used as initial guesses to search for the subset shape function parameters corresponding to the image-points matched at time $t_{0}+\Delta_{t}$.

$\overline{t_{0}+\Delta_{t-1}}$ and $t_{0}+\Delta_{t}$ correspond to two consecutive acquisition instants. 


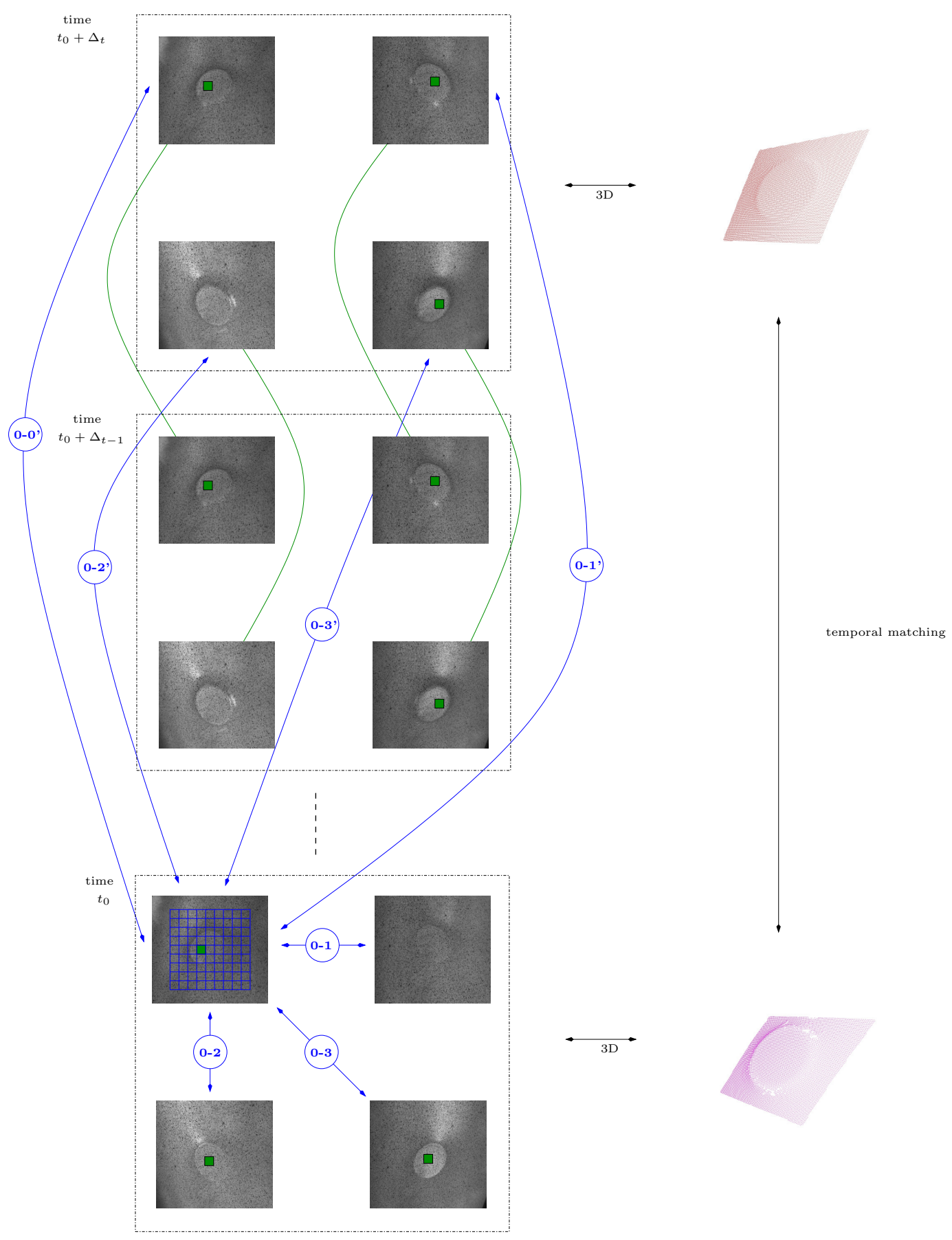

Fig. 12. Methodology for processing three sets of four images taken at three different instants $\left(t_{0}, t_{0}+\Delta_{t-1}\right.$ and $\left.t_{0}+\Delta_{t}\right)$ corresponding to two different levels of deformation of the part. Time $t_{0}$ corresponds to the initial state and $t_{0}+\Delta_{t-1}$ and $t_{0}+\Delta_{t}$ correspond to two consecutive acquisition instants. 


\subsection{Results (experiment II)}

The methodology described in the previous section has been applied during the Single Point Incremental Forming of a part (experiment II).

First, the 4-camera system has been calibrated using the method described in Section 3. The calibration accuracy was very good (see Section 3.2 and Table 4).

After the calibration step, a sequence of 4-images has been acquired during the Single Point Incremental Forming of the part.

The sequence of 4-images (image \#0 to image \#3) has been processed using our method (see Figure 12) leading to a sequence of displacement fields.

Figure 13 shows the 3D shape of the formed part computed by our method at two different instants $t$ and $t+\Delta_{t}$.

Figure 14 shows the displacement map (norm of the displacement vector) between time $t$ and time $t+\Delta_{t}$. 


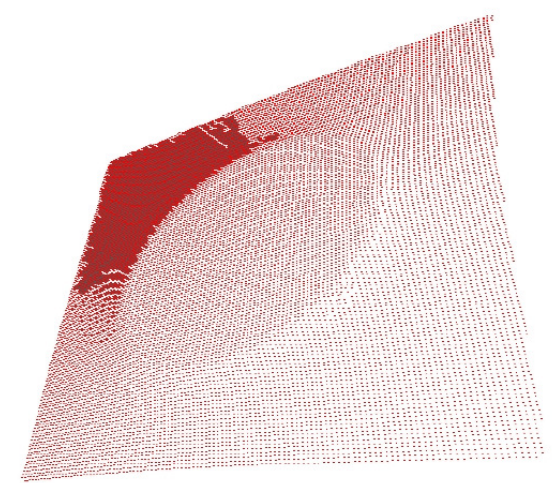

(a)

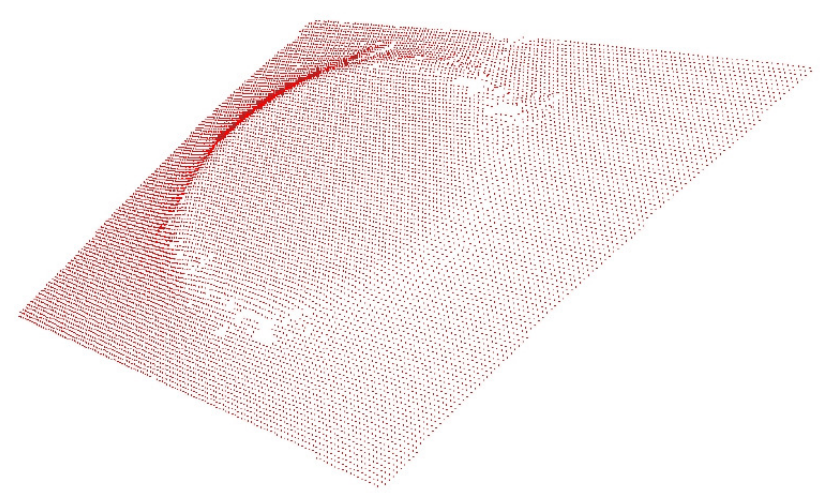

(b)

Fig. 13. 3D shape of the formed part at time $t$ (a) and at time $t+\Delta_{t}(\mathrm{~b})$. 


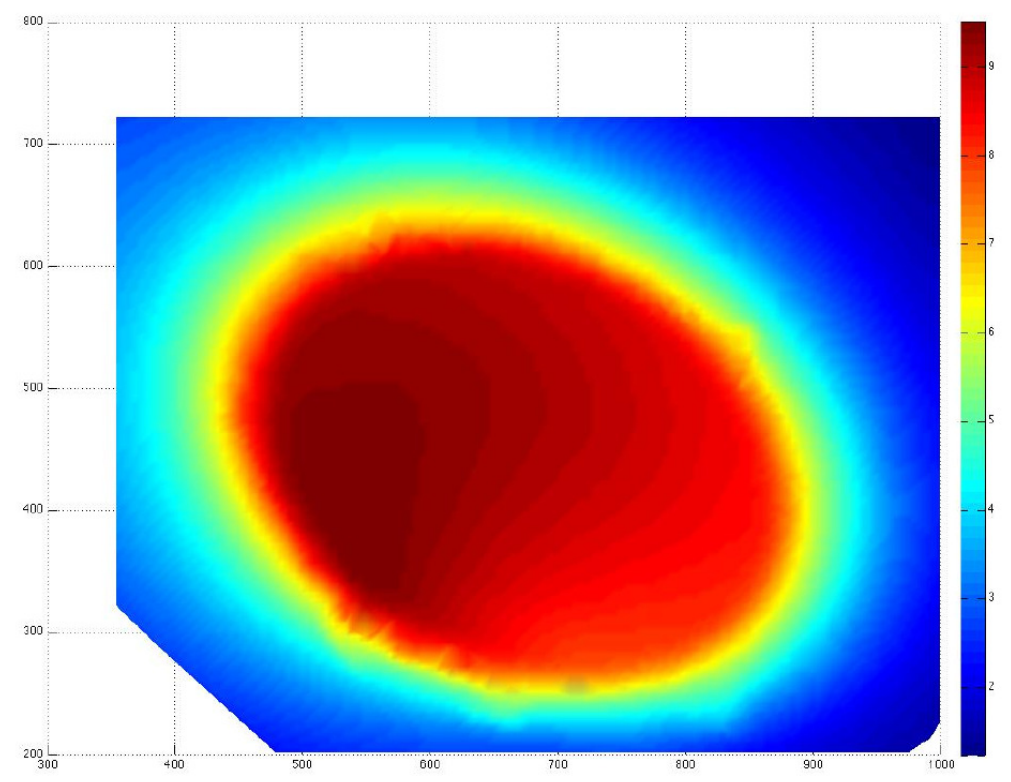

Fig. 14. Displacement map (norm of the displacement vector in $\mathrm{mm}$ ) between time $t$ and time $t+\Delta_{t}$. 


\begin{tabular}{|l|c|c|}
\hline & camera 0 & camera 1 \\
\hline$c_{x}$ (pixel) & $686.2 \pm 30.22$ & $687.9 \pm 36.68$ \\
\hline$c_{y}$ (pixel) & $534.1 \pm 40.17$ & $508.3 \pm 48.79$ \\
\hline$f_{x}$ (pixel) & $3574.9 \pm 13.89$ & $3599.4 \pm 13.65$ \\
\hline$f_{y}$ (pixel) & $3574.9 \pm 13.89$ & $3599.4 \pm 13.65$ \\
\hline$d_{1}$ & $-0.345 \pm 1.01 \mathrm{e}-05$ & $-0.353 \pm 1.32 \mathrm{e}-05$ \\
\hline
\end{tabular}

Standard deviation of residuals for all views (23 views): 0.023 pixels

Table 5

Calibration of the stereovision system with Vic-3D ${ }^{\circledR}$ : intrinsic parameters of experiment II (1st radial distortion model with skew factor set to 0 ).

\subsection{Validation and discussion}

In order to validate our results, the $3 \mathrm{D}$ displacement field of the part surface has also been computed using the well-established stereovision method ( 2 cameras). The Vic-3D ${ }^{\circledR}$ stereovision software [7] has been used.

Before starting the forming (during the calibration of the 4-camera system), two cameras among the 4-camera system (camera \#0 and camera \#1 on Figure 12) have been calibrated using the Vic-3D ${ }^{\circledR}$ software. The calibration parameters are given in Table 5. The calibration accuracy was very good (standard deviation of residuals for all views: 0.023 pixels).

The sequence of stereo pair of images (image \#0 and image \#1) extracted from the 4-images sequence has been processed using the Vic-3D ${ }^{\circledR}$ software leading to a sequence of displacement fields. It should be noted that the same image-points have been processed by the two methods (our method and the Vic-3D ${ }^{\circledR}$ method) as the same initial 2D mesh and the same seed point have been used in both methods. 
Figure 15-(a) shows the displacement map (norm of the displacement vector) computed by the stereovision method between time $t$ and time $t+\Delta_{t}$.

Figure 15-(b) shows the point-to-point absolute difference between the two displacement maps (shown on Figures 14 and Figures 15-(a)). This figure shows that the discrepancy between both maps is very small, except in some specific areas. The mean is $0.10 \mathrm{~mm}$ and the standard deviation is $0.071 \mathrm{~mm}$, with a max value of $1.12 \mathrm{~mm}$.

As it is visible on Figure 15-(b), the result is corrupted by some points for which the discrepancy is higher than $1 \mathrm{~mm}$. These points, that are located on the slopes of the formed part, correspond to image-points that have been badly correlated during the DIC process. A detailed observation of the images shows that they suffer from high specular reflection in these areas.

In order to remove these bad points from the statistical analysis, the absolute difference between both maps has been computed on the rectangular area shown on Figure 15-(b) (center of the figure corresponding to the top of the formed part). A mean of $0.15 \mathrm{~mm}$, a standard deviation of $0.01 \mathrm{~mm}$ and a max of $0.18 \mathrm{~mm}$ have been obtained.

It should be noted that the badly correlated image-points could be probably filtered by using the epipolar constraint into the DIC process. An image-point should be eliminated if its distance to its corresponding epipolar line is higher than a threshold. This functionality is being implemented in our software and this will probably improve the quality of the $3 \mathrm{D}$ reconstruction by eliminating some false matches at the DIC level. 


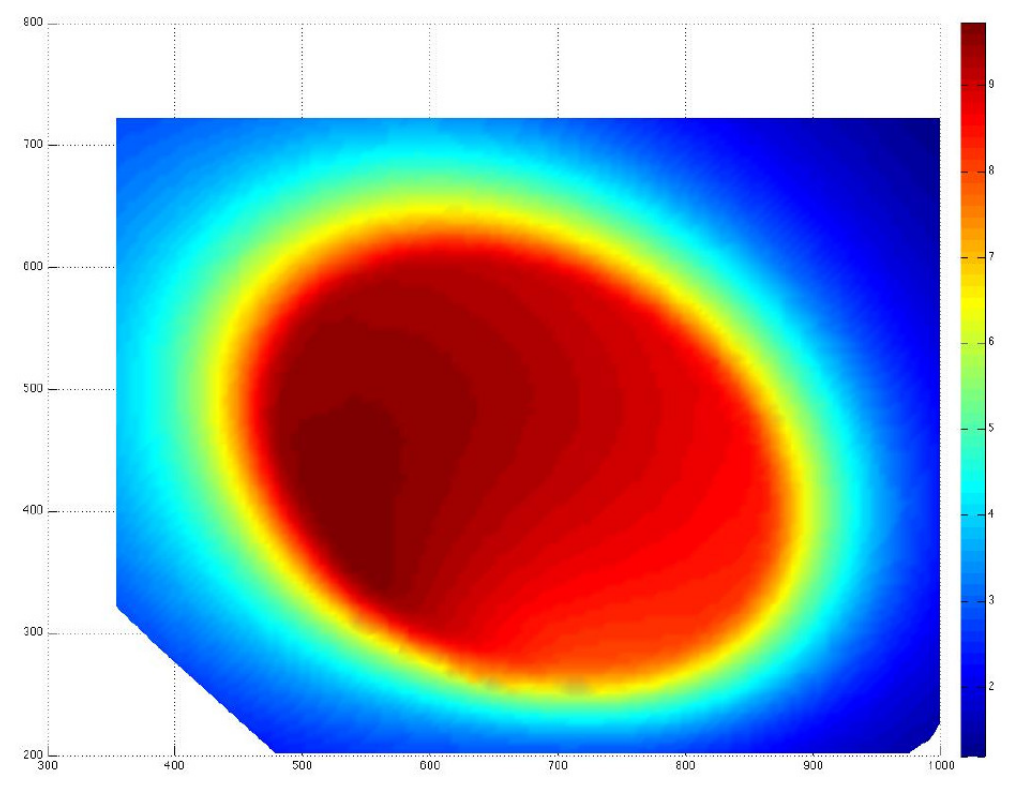

(a)

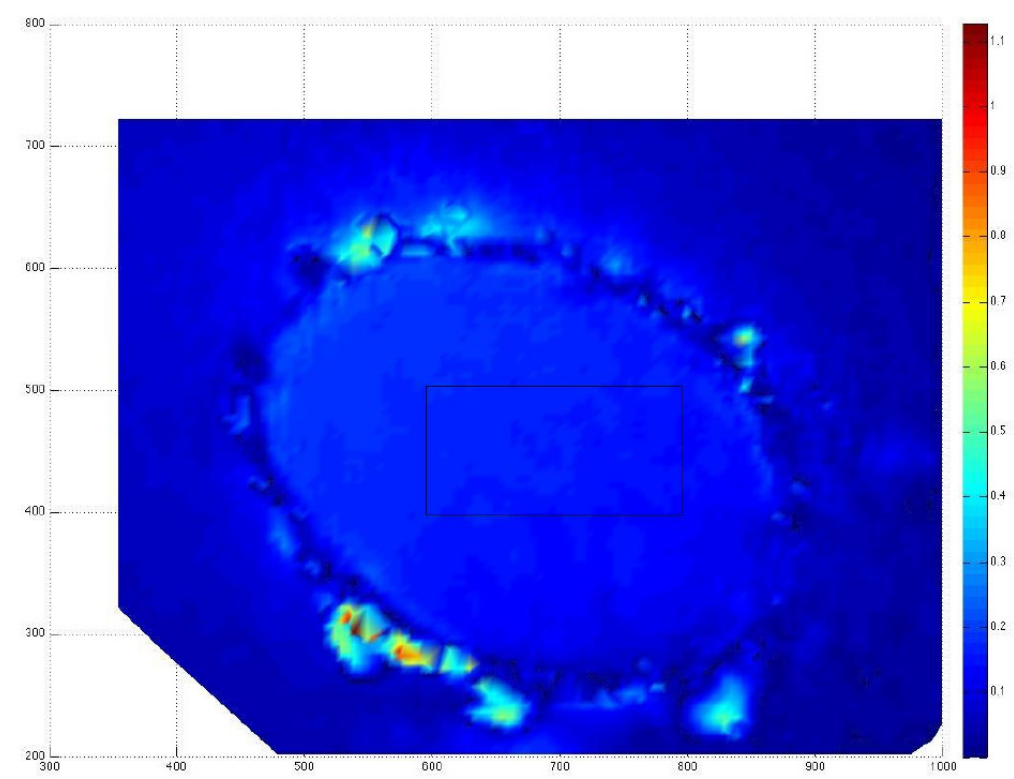

(b)

Fig. 15. (a) Displacement map (norm of the displacement vector in $\mathrm{mm}$ ) computed with Vic-3D ${ }^{\circledR}$ and (b) point-to-point absolute difference between the two displacement maps. The rectangular area in the center of Figure (b) has been used to compute some statistical values on the top of the formed part. 


\section{Conclusion}

A multiple-camera system (four cameras in the present study) has been developed to measure the shape variations and the 3D displacement field of a sheet metal part during a Single Point Incremental Forming (SPIF) operation.

The multiple-camera system is calibrated using a Sparse Bundle Adjustment method based on the Levenberg-Marquardt algorithm and it is shown that an accurate calibration can be achieved.

The multiple-image matching is performed using DIC.

Such a system enables to observe the part surface from different view points and provides directly the 3D shape (one-shot image acquisition) or the 3D displacement field of the whole part.

Our approach does not require any 3D stitching operation, nor the use of markers, as the multiple-camera system provides directly all the 3D measurements in a common coordinate system. Any reasonable number of cameras (even an odd number of cameras) can be managed ${ }^{7}$.

Two experiments have been carried out (one for Shape Measurement and one for 3D Displacement Measurement) and the quality of the results has been demonstrated.

Some areas of improvement are currently being investigated such as the use of the epipolar constraint into the DIC process to filter some false matches or the use of a wand-based calibration method [27,28] (as shown on Figure 5, we are currently using a plane-based calibration method) that would be more adapted to some multiple-camera configurations since, with one-dimensional calibration objects, the calibration point(s) can be observed by all the cameras

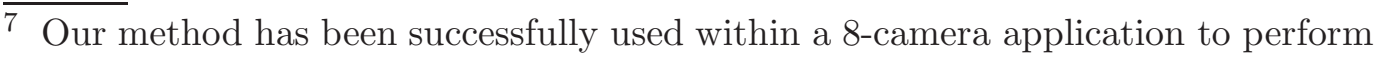
the NDT inspection of large aeronautical structures [8-10]. 
at the same time.

\section{Acknowledgments}

We thank Didier Adé for his helping us carry out the experiments.

\section{A Modeling and Calibration of a Single Camera}

In this Appendix, the modeling and the calibration of a single camera is presented in order to introduce the general methodology of our approach that has been extended to the modeling and calibration of a multiple-camera system (see Section 3).

A classical pinhole camera model (linear model) is used. A set of distortion parameters is added to this model to take into account the optical distortion of the lens (the model becomes non linear) [26].

As shown in Figure A.1, we denote $\mathcal{R}_{\mathrm{w}}$ the world coordinate system, $\mathcal{R}_{\mathrm{c}}$ the camera coordinate system (which has its origin at the lens center), $\mathcal{R}_{\mathrm{r}}$ the retinal coordinate system associated to the sensor plane and $\mathcal{R}_{\mathrm{s}}$ the image coordinate system (in pixel units).

The transformation that maps any $3 \mathrm{D}$ point $M$ in $\mathcal{R}_{\mathrm{w}}$ into a $2 \mathrm{D}$ image-point $m$ (in pixel units) consists of four consecutive transformations:

(1) a transformation between $\mathcal{R}_{\mathrm{w}}$ and $\mathcal{R}_{\mathrm{c}}$. This is a rigid-body transformation (rotation $\mathbf{R}+$ translation $\mathbf{t}) \mathbf{L}=(\mathbf{R}, \mathbf{t})$, that transforms a $3 \mathrm{D}$ point $M$ in $\mathcal{R}_{\mathrm{w}}$ into a $3 \mathrm{D}$ point $M_{c}$ in $\mathcal{R}_{\mathrm{c}}: M_{c}=\mathbf{L}(M)$

(2) a transformation between $\mathcal{R}_{\mathrm{c}}$ and $\mathcal{R}_{\mathrm{r}}$. This is a projective transformation $\mathbf{P}$ (imaging process) that transforms the $3 \mathrm{D}$ point $M_{c}$ into a $2 \mathrm{D}$ point $m_{r}$ in the retinal plane: $m_{r}=\mathbf{P}\left(M_{c}\right)$ 
(3) a transformation between $\mathcal{R}_{\mathrm{r}}$ and $\mathcal{R}_{\mathrm{s}}$. This transformation describes the sampling of the intensity field incident on the sensor array. A $2 \mathrm{D}$ point $m_{r}$ in the retinal plane (in metric units) is transformed into a 2D undistorted point $m$ in the sensor image plane (in pixel units): $\tilde{m}=\mathbf{K}_{\mathbf{k}}\left(m_{r}\right)$.

This transformation depends on the five camera intrinsic parameters $\mathbf{k}=$ $\left(c_{x}, c_{y}, f_{x}, f_{y}, s\right)$, where $c_{x}$ and $c_{y}$ are the coordinates of the principal point (in pixel units), given by the intersection of the optical axis with the retinal plane, $f_{x}$ and $f_{y}$ are the focal length in pixels in the $x$ and $y$ direction, and $s$ is the skew factor.

(4) a transformation $D_{d}$ that transforms an ideal undistorted pixel into a distorted pixel: $m=\mathrm{D}_{\mathbf{d}}(\tilde{m})$.

This transformation depends on several distortions parameters ${ }^{8}$. The number of distortion parameters (typically from 1 to 7 ) depends on the distortion model that is used [26]. In the sequel, we will suppose that a 3-parameter-distortion-model is used (3rd order radial distortion model).

The composition of these four transformations can be written:

$$
\begin{aligned}
f: \mathbb{R}^{5} \times \mathbb{R}^{3} \times \mathbb{R}^{3} \times \mathbb{R}^{3} \times \mathbb{R}^{3} \longmapsto & \mathbb{R}^{2} \\
(\mathbf{k}, \mathbf{d}, \mathbf{R}, \mathbf{t}, M) & \longmapsto m=\mathrm{D}_{\mathbf{d}}\left(\mathbf{K}_{\mathbf{k}}(\mathbf{P}(\mathbf{L}(M)))\right)
\end{aligned}
$$

and leads to the following equation that relates a 3D point in space $M$ and its $2 \mathrm{D}$ projection $m$ onto the camera sensor array (pixel coordinates) :

$$
m=\mathrm{f}(\mathbf{k}, \mathbf{d}, \mathbf{R}, \mathbf{t}, M)=\mathrm{f}_{\mathbf{k}, \mathbf{d}, \mathbf{L}}(M)
$$

Calibrating the camera consists in determining its parameters $\mathbf{k}, \mathbf{d}, \mathbf{R}$ and t. The experimental calibration procedure generally uses a calibration target that provides known $3 \mathrm{D}$ points. In our work, the calibration is performed by acquiring a series of $n$ images of a planar calibration target made up of $p$ cir-

8 Note that the distortion parameters are also intrinsic to the camera and they could be added to the intrinsic parameters vector. 
cular points, observed at different positions and orientations (see Figure A.2). Thus, the calibration target provides a set of $p$ known 3D points.

We call $m_{i}^{j}$ the $2 \mathrm{D}$ projection into the camera image plane of the $j^{\text {th }} 3 \mathrm{D}$ point $M_{j}(j=1 \ldots p)$ of the $i^{\text {th }}$ view $(i=1 \ldots n)$. We define a set of localization matrices $\mathbf{L}_{i}=\left(\mathbf{R}_{i}, \mathbf{t}_{i}\right)(i=1 \ldots n)$ that represent the $i^{\text {th }}$ position of the calibration target with respect to camera reference frame (see Figure A.2).

The estimation of the parameters in the least-square sense leads to a non-linear optimization process, where the sum of the 2D Euclidean distance between the estimated projection of the $j$-th point of the $i$-th view onto the camera (using Eq. (A.2)) and the actual image-point $m_{i}^{j}$ extracted in the $i$-th image is minimized:

$$
\theta=\arg \min _{\theta} \sum_{i=1}^{n} \sum_{j=1}^{p}\left\|m_{i}^{j}-\mathrm{f}\left(\mathbf{k}, \mathbf{d}, \mathbf{R}_{i}, \mathbf{t}_{i}, M_{j}\right)\right\|^{2}
$$

where $\theta=\left(\mathbf{k}, \mathbf{d}, \mathbf{R}_{1} \ldots \mathbf{R}_{n}, \mathbf{t}_{1} \ldots \mathbf{t}_{n}, M_{1} \ldots M_{p}\right)$ is the vector of the $8+6 n+$ $3 p$ parameters that are estimated during the minimization process. It should be noted that there are $2 n p$ equations (each $3 \mathrm{D}$ point projection gives two relations). With a judicious choice of $n$ and $p$, there are more equations than unknowns (over-determined system).

The estimation of the camera intrinsic parameters $\mathbf{k}$ and $\mathbf{d}$, the extrinsic parameters $\mathbf{R}_{i}$ and $\mathbf{t}_{i}$ of each view, and the $3 \mathrm{D}$ points $M_{j}$ of the calibration target is a well-known problem referred to as Bundle Adjustment [25, 29]. It is solved using the Levenberg-Marquardt algorithm. In order the algorithm to converge, an initial guess of the parameters is required. An initial guess of the 5 intrinsic parameters (vector $\mathbf{k}$ ) and of the extrinsic parameters related to each view $\left(\mathbf{R}_{i}\right.$ and $\left.\mathbf{t}_{i}\right)$ is computed using the closed-form solution described in [30] or [31]. The distortion parameters (vector $\mathbf{d}$ ) are simply initialized to 0. An initial guess of the 3D points $\left(M_{j}\right)$ is given by the user supplied model of the target. As the points of the target will be re-estimated, the initial model need not be known accurately which is a great advantage of this calibration method. 


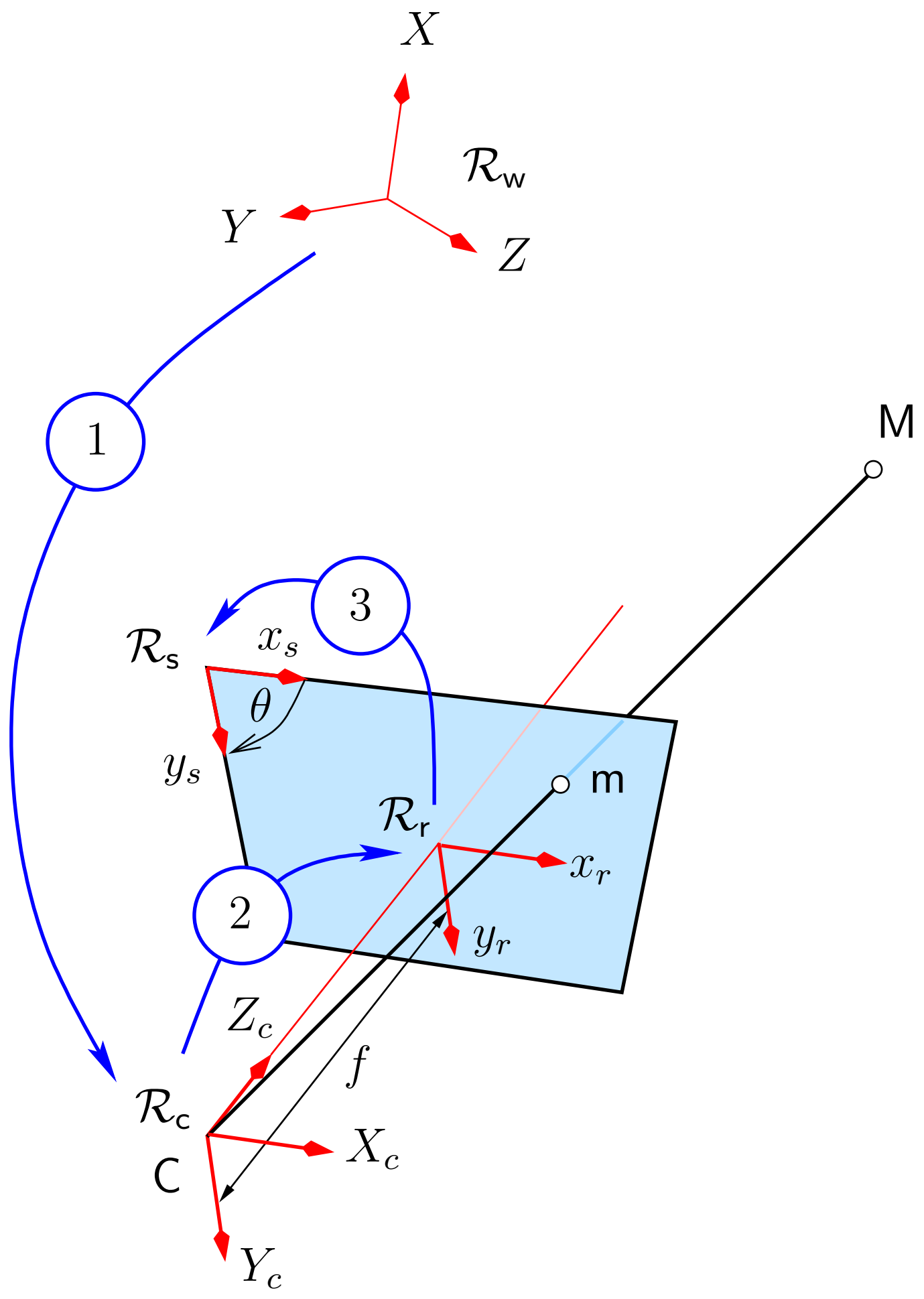

Fig. A.1. The three elementary transformations of the pinhole camera model, and the associated coordinate systems. The first transformation relates the coordinate of a scene point into the camera coordinate system. The second one is the projection transformation of this point onto the retinal plane. The third one transform the point into the sensor coordinate system (undistorted pixel). 


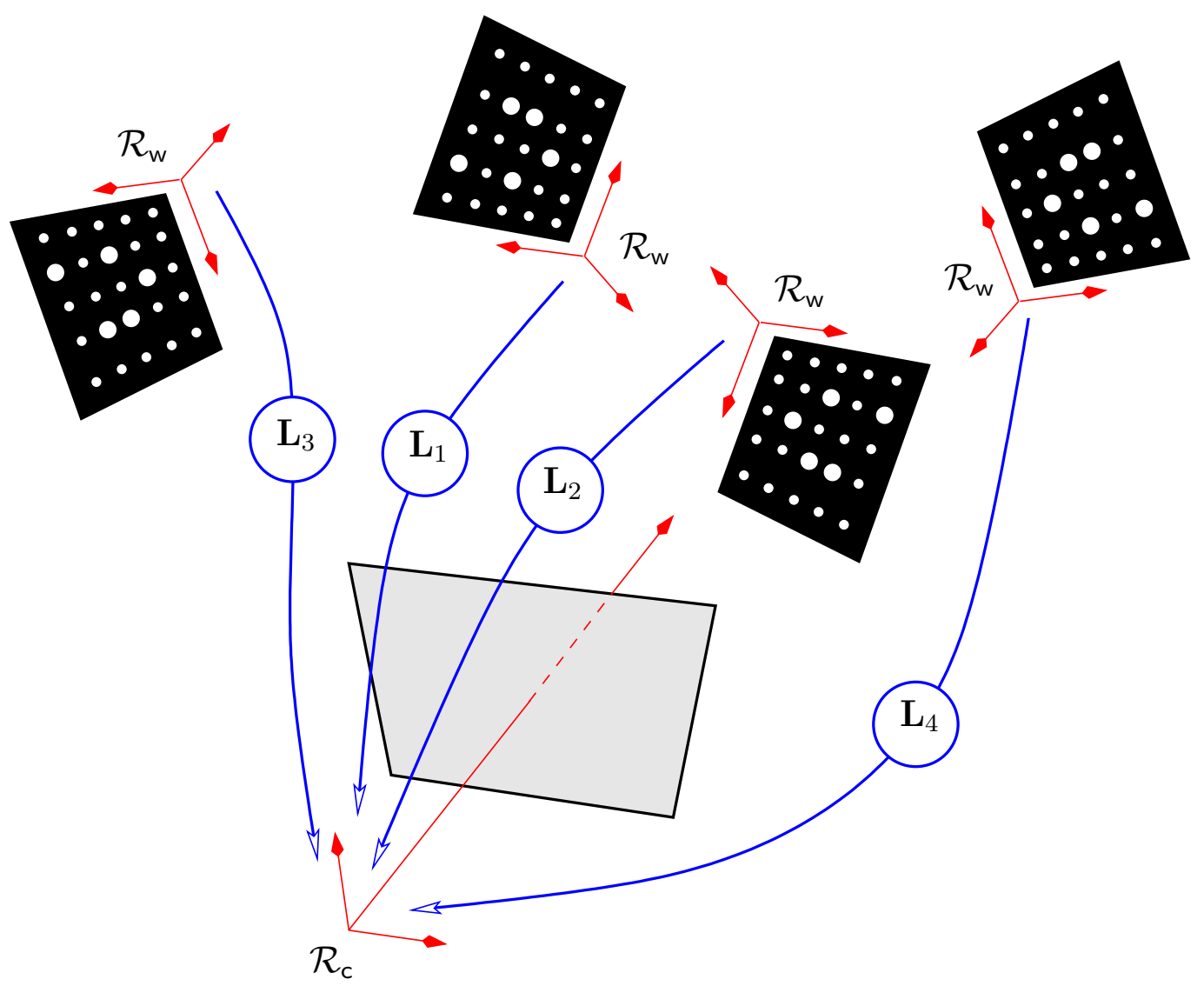

Fig. A.2. Procedure for calibrating a single camera: a series of images of a planar calibration target observed at different positions and orientations is acquired by the camera. 


\section{References}

[1] Jeswiet J., Micari F., Hirt G., Bramley A., Duflou J., and Allwood J. (2005) Asymmetric Single Point Incremental Forming of Sheet Metal. CIRP Annals Manufacturing Technology, 54(2):88-114.

[2] Jeswiet J., Young D., and Ham M. (2005) Non-Traditional Forming Limit Diagrams for Incremental Forming. Advanced Materials Research, 6-8:409-416.

[3] Shim M. S. and Park J. J. (2001) The formability of aluminium sheet in incremental forming. Journal of Materials Processing Technology, 113:654-658.

[4] Micari F., Ambrogio G., and Filice L. (2007) Shape and dimensional accuracy in Single Point Incremental Forming: State of the art and future trends. Journal of Materials Processing Technology, 191:390-395.

[5] Kim T. J. and Yang D. Y. (2000) Improvement of formability for the incremental sheet metal forming process. International Journal of Mechanical Sciences, 42:1271-1286.

[6] He S., Van Bael A., Van Houtte P., Tunckol Y., Duflou J., Henrard C., Bouffioux C., and Habraken A. M. (2005) Effect of FEM choices in the modelling of incremental forming of aluminium sheets. Proceedings of the 8th ESAFORM Conference, Cluj-Napoca, Romania.

[7] Vic-Snap ${ }^{\circledR}$ and Vic-3D ${ }^{\circledR} \quad$ (2008), Correlated Solutions Inc., http://www. correlatedsolutions.com/.

[8] Harvent J., Bugarin F., Orteu J.-J., Devy M., Barbeau P., and Marin G. (2008) Inspection of aeronautics parts for shape defect detection using a multi-camera system. Proceedings of SEM XI International Congress on Experimental and Applied Mechanics, Orlando, FL, USA, 2-5 June.

[9] Bugarin F., Decultot N., Devy M., Harvent J., Orteu J.-J., Robert L., and Velay V. (2008) Multi-camera computer vision for large volume inspection: (a) aeronautical parts inspection and (b) incremental forming. Large Volume Metrology Conference 2008 (LVMC'2008), Liverpool, UK, 4-5 November, available from http://www. Ivmc.org.uk/2008_presentations.shtml. 
[10] Harvent J. (2010) Mesure de formes par corrélation multi-images : application à l'inspection de pièces aéronautiques à l'aide d'un système multi-caméras. Ph.D. thesis, Université de Toulouse, France.

[11] Robert L., Decultot N., Velay V., and Bernhart G. (2008) Behaviour identification of aluminium sheet in single point incremental forming by finite element method updated. Photomechanics'2008, Loughborough, UK, 7-9 July.

[12] Sutton M. A., Orteu J.-J., and Schreier H. W. (2009) Image Correlation for Shape, Motion and Deformation Measurements - Basic Concepts, Theory and Applications. 364 p. 100 illus. Hardcover, Springer, ISBN 978-0-387-78746-6.

[13] Sutton M. A., Wolters W. J., Peters W. H., and McNeill S. R. (1983) Determination of displacements using an improved digital correlation method. Image and Vision Computing, 1:133-139.

[14] Vasilakos I., Gu J., Belkassem B., Sol H., Verbert J., and Duflou J. R. (2009) Investigation of Deformation Phenomena in SPIF Using an In-Process DIC Technique. Key Engineering Materials, 410-411:401-409.

[15] Sasso M., Callegari M., and Amodio D. (2008) Incremental forming: an integrated robotized cell for production and quality control. Meccanica, 43:153163.

[16] Faugeras O. (1993) Three-Dimensional Computer Vision: A Geometric Viewpoint. The MIT Press, ISBN 0-262-06158-9.

[17] Hartley R. I. and Zisserman A. (2004) Multiple View Geometry in Computer Vision. Cambridge University Press, ISBN: 0521540518, second edn.

[18] Garcia D., Orteu J.-J., and Devy M. (2000) Accurate Calibration of a Stereovision Sensor: Comparison of Different Approaches. Proceedings of 5th Workshop on Vision, Modeling, and Visualization (VMV'2000), Saarbrücken (Germany), 22-24 November, pp. 25-32.

[19] Garcia D. (2001) Mesure de formes et de champs de déplacements tridimensionnels par stéréo-corrélation d'images. Ph.D. thesis, Institut National Polytechnique de Toulouse, France. 
[20] Lourakis M. I. A. and Argyros A. A. (2004) The design and implementation of a generic sparse bundle adjustment software package based on the levenberg$\begin{array}{llll}\text { marquardt } & \text { algorithm. } & \text { Tech. }\end{array}$ Institute of Computer Science - FORTH, Heraklion, Crete, Greece, available from http://www.ics.forth.gr/ lourakis/sba.

[21] Ayache N. (1991) Artificial Vision for Mobile Robots - Stereo-Vision and Multisensory Perception. The MIT Press, ISBN 978-0-262-01124-2.

[22] Garcia D., Orteu J.-J., and Penazzi L. (2002) A combined temporal tracking and stereo-correlation technique for accurate measurement of 3D displacements: application to sheet metal forming. Journal of Materials Processing Technology, 125-126:736-742.

[23] Orteu J.-J., Cutard T., Garcia D., Cailleux E., and Robert L. (2007) Application of Stereovision to the Mechanical Characterisation of Ceramic Refractories Reinforced with Metallic Fibres. Strain, 43(2):96-108.

[24] Orteu J.-J. (2009) 3-D Computer Vision in Experimental Mechanics. Optics and Lasers in Engineering, 47(3-4):282-291.

[25] Triggs B., McLauchlan P. F., Hartley R. I., and Fitzgibbon A. W. (2000) Bundle Adjustment - A Modern Synthesis. ICCV'99: Proceedings of the International Workshop on Vision Algorithms, London, UK, pp. 298-372, Springer-Verlag.

[26] Weng J., Cohen P., and Herniou M. (1992) Camera Calibration with Distorsion Models and Accuracy Evaluation. Pattern Analysis and Machine Intelligence (PAMI), 14(10):965-980.

[27] Zhang Z. (2004) Camera Calibration with One-Dimensional Objects. IEEE Transactions on Pattern Analysis and Machine Intelligence, 26(7):892-899.

[28] Pribanić T., Sturm P., and Peharec S. (2009) Wand-Based Calibration of 3D Kinematic System. IET Computer Vision, 3:124-129.

[29] Lavest J.-M., Viala M., and Dhome M. (1998) Do we really need an accurate calibration pattern to achieve a reliable camera calibration? Proceedings of the 5th European Conference on Computer Vision (ECCV'98) - Volume I, London, UK, pp. 158-174, Springer-Verlag. 
[30] Ravn O., Andersen N. A., and Sorensen A. T. (1993) Auto-calibration in Automation Systems using Vision. 3rd International Symposium on Experimental Robotics (ISER'93), (Japon), pp. 206-218.

[31] Zhang Z. (2000) A Flexible New Technique for Camera Calibration. IEEE Transactions on Pattern Analysis and Machine Intelligence, 22(11):1330-1334. 